\title{
Effects of artificial light on bird movement and distribution: a systematic map
}

\author{
Carrie Ann Adams ${ }^{1 *} \mathbb{0}$, Esteban Fernández-Juricic ${ }^{2}$, Erin Michael Bayne ${ }^{1}$ and Colleen Cassady St. Clair ${ }^{1}$
}

\begin{abstract}
Background: Artificial light is ubiquitous in the built environment with many known or suspected impacts on birds. Birds flying at night are known to aggregate around artificial light and collide with illuminated objects, which may result from attraction and/or disorientation. In other contexts, birds are repelled by light-based deterrents, including lasers and spotlights. Artificial light can also change birds' perceptions of habitat quality, resulting in selection or avoidance of illuminated areas. Studies documenting aggregation, deterrence, and habitat selection are typically considered separate literature bodies, but they actually study a common set of populations, interventions/exposures, and responses. Our systematic map provides a comprehensive, searchable database of evidence of the effects of artificial light on bird movement and distribution, increasing both the quantity and diversity of studies that are accessible for further comparison and synthesis. We identify and describe the evidence available for four secondary questions relevant to conservation or management: aggregation/mortality at structures with artificial lights, evidence that light attracts and/or disorients birds, light-based deterrent efficacy, and the influence of continuous illumination on habitat selection.
\end{abstract}

Methods: Using the principles of systematic reviews and methods published in an earlier protocol, we conducted an extensive and interdisciplinary literature search. We searched multidisciplinary citation indices as well as databases and websites specific to conservation, pest management, transportation, and energy. In our map, we included all studies reporting eligible populations (birds), interventions/exposures (artificial light), and outcomes (movement through space, behaviour preceding movement, or distribution). We evaluated the quantity of available evidence based on meta-data fields related to study context, population traits, light source characteristics, and outcome variables. We used these meta-data to identify relevant evidence for each secondary question and describe aspects of our secondary questions that may support reviews (evidence clusters) and others that require more research (knowledge gaps).

Review findings: We manually screened 26,208 articles and coded meta-data for 490 eligible studies in a searchable database, organizing the literature to facilitate future reviews and evidence-based management. Much of the evidence was concentrated in particular locations (Northern hemisphere), taxonomic orders (Passeriformes, Charadriiformes, and others), and light wavelengths (red and white). We identified 56 distinct response variables and organized them into 3 categories (behaviour, distribution, and avian community), showing the diversity in bird responses to light.

Conclusions: Our database can be used to answer the secondary questions we identified and other questions about the effects of artificial light on bird movement and resulting changes to distribution. There may be sufficient evidence

\footnotetext{
*Correspondence: caadams1@ualberta.ca

${ }^{1}$ Department of Biological Science, University of Alberta, CW 405,

Biological Sciences Building, Edmonton, AB T6G 2E9, Canada

Full list of author information is available at the end of the article
}

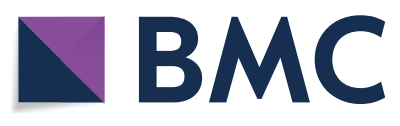

(c) The Author(s) 2021. Open Access This article is licensed under a Creative Commons Attribution 4.0 International License, which permits use, sharing, adaptation, distribution and reproduction in any medium or format, as long as you give appropriate credit to the original author(s) and the source, provide a link to the Creative Commons licence, and indicate if changes were made. The images or other third party material in this article are included in the article's Creative Commons licence, unless indicated otherwise in a credit line to the material. If material is not included in the article's Creative Commons licence and your intended use is not permitted by statutory regulation or exceeds the permitted use, you will need to obtain permission directly from the copyright holder. To view a copy of this licence, visit http://creativecommons.org/licenses/by/4.0/. The Creative Commons Public Domain Dedication waiver (http://creativeco mmons.org/publicdomain/zero/1.0/) applies to the data made available in this article, unless otherwise stated in a credit line to the data. 
for a review of the weather and lunar conditions associated with collisions, which could help identify nights when reduction of artificial light is most important. Further experiments should investigate whether specific types of light can reduce collisions by increasing the detectability of structures with artificial lights. The efficacy of lasers as deterrents could be evaluated through systematic review, though more studies are needed for UV/violet lasers. To reduce the impacts of outdoor lighting on birds, research should investigate how spectral composition of white light influences bird attraction, orientation, and habitat selection.

\section{Introduction}

\section{Background and objectives}

Artificial light is growing worldwide [1], with diverse biological and ecological impacts [2]. Electric light sources have different spectral properties and higher intensities than celestial light, and both electric lighting and anthropogenic flames change the daily, monthly, and annual light cycles under which most organisms evolved [3]. Artificial light at night (ALAN) can alter daily activity patterns and affect organismal physiology, particularly hormone levels and stress responses [2]. In some species, ALAN elicits positive phototaxis $[4,5]$ or interferes with orientation and navigation [6,7], limiting dispersal [8] and changing broad scale distributions [9]. Phototaxis can lead directly to mortality in sea turtles [10], insects [5], and fledgling seabirds [11], and may have sublethal impacts that are more difficult to quantify [9]. ALAN can also affect habitat selection $[6,12,13]$ and population metrics including abundance [14-16] and reproduction [5], potentially affecting population trajectories [17-19]. The strength and direction of the effects of ALAN varies widely among species, creating imbalances that disrupt trophic relationships and pollination networks [20-24]. Most research has focused on artificial light at night, but daytime artificial lighting can also have ecological impacts and management applications if it: (a) illuminates places that are otherwise dark [25]; (b) uses wavelengths that are lethal or harmful to particular species [26]; or (c) creates a novel stimuli that may act as an attractant or activate an organisms' anti-predator response (e.g. light-based deterrents) [27].

For birds, most well-known effects of artificial light occur during nocturnal migration, when birds are frequently observed aggregating around or colliding with structures with artificial light, such as communication towers and their associated guy wires [28, 29]. Birds aggregate around beams of light projected into the sky, as seen at ceilometers and at the September $11^{\text {th }}$ memorial, diverting, delaying, and sometimes grounding them during migration (e.g. [30, 31]). Groundings have been documented extensively for fledgling seabirds at coastal light sources [11]. These aggregations present a conservation concern because artificial light has been associated with all major sources of collision mortality: buildings and windows, transmission lines, roads, and communication towers [32].

The proximate and ultimate causes of these aggregations remain unclear. Many authors attribute these aggregations to birds preferentially flying towards light sources, which would suggest attraction to them [31, 33]. Even if they do not preferentially fly towards light sources, birds may be unwilling to leave an illuminated area once they encounter it in their flight path, a phenomenon called capture [28, 34, 35]. Attraction could be caused by an overstimulation of the visual system [36]. Artificial light becomes polarized when reflected off of asphalt surfaces, which may attract waterbirds who mistake the asphalt for water and become injured or stranded [37]. This interpretation assumes that birds see polarized light, which is controversial [38]. Additionally, birds may fly towards light sources to increase visibility during flight or enhance the chances of detecting predators.

Most authors agree that birds aggregating around light sources are disoriented; they have lost the ability to select and maintain a certain direction [39]. Artificial light has the potential to disrupt each of the three primary mechanisms of compass orientation in migratory birds: solar, stellar, and magnetic. Birds use the position of the sun to orient during the day and at dusk, taking the time-of-day and day-of-year into account $[39,40]$. Clock-shift experiments have shown that some birds orient in the wrong direction when exposed to daylight periods shifted by several hours, even when daylength matches the local photoperiod (e.g. [40-42]). It is unknown whether outdoor lighting causes birds in the wild to perceive clockshifts of sufficient magnitude to disorient them. Artificial light may also interfere with the stellar compass, which forms when young birds identify the center of celestial rotation during their first spring and learn to use constellations to orient north and south [39, 43, 44]. There is individual variation in which and how many stars they require to orient [45]. The night sky birds encounter during migration could be very different than the stellar patterns they learned if skyglow levels change or increasing numbers of satellites move across the night sky, as is expected within the next decade [46-48]. During magnetic compass orientation, a radical-pair process 
in the retina allows birds to detect the inclination of the earth's magnetic field and specific types of artificial light interfere with this ability [49]. If artificial light bleaches the rhodopsin in their retina, birds could lose their ability to see relatively dim light coming from the stars and the reflection of dim celestial light off of landmarks [50]. Disorientation can explain the loss of a linear flight path, though it is unclear why disoriented birds remain near the artificial light sources.

In contrast to the examples of apparent attraction, light has also been used to deter birds from zones of humanwildlife conflict. A better understanding of deterrent efficacy is necessary to prevent millions of dollars in damage to crops and aircraft $[51,52]$. Birds are assumed to respond to approaching vehicles as they would to a predator [53], and artificial light is used to increase the detectability and perceived risk of the vehicle, increasing detection, alert and flight initiation distances [54, 55]. To elicit an avoidance response without an approaching object, light-based deterrents must create the illusion of risk that outweighs the benefits of using a resource (e.g. food). Deterring birds can be especially challenging when there is no immediate risk to the bird (e.g. roost trees in cities), the birds may not perceive the risk (e.g. toxic tailings ponds), or there are significant attractants (e.g. aquaculture ponds). If non-lethal deterrents fail, continued conflict may result in lethal management strategies. Ultimately, interventions to reduce bird attraction to artificial light or increase bird deterrence using light-based interventions have the same goal-to change bird movement and distribution.

Understanding how light changes bird distributions is important because it is often the goal of interventions using or reducing artificial light, but it is also sometimes the metric by which the ecological impacts of exposure to artificial light are measured. Bird distributions result from habitat selection, the process of choosing resources through a series of innate or learned behavioural decisions [56]. Studies of bird distributions, including population density or locations of individuals, are often used to infer habitat selection and identify important habitat components in conservation planning under the assumption that species have evolved to select higher quality habitat $[57,58]$. Artificial light can influence habitat quality in diverse ways that include changes to diel activity patterns [59, 60], hormone production [61], cognitive function [62, 63], and nestling development [64-66]. Artificial light can also change the relative abundance of bird prey and predators [67-69], affecting the chances of survival and reproduction. Even when artificial light seems to increase bird abundance or use of illuminated areas, there may not be corresponding increases in reproductive success, particularly in human-modified landscapes [70]. Habitat selection may also be driven by innate attraction to or avoidance of light [71]. Birds may adequately perceive some impacts of artificial light on habitat quality (e.g. changes to prey abundance) and respond with selection decisions that improve fitness, while other impacts could be more difficult for both birds and human researchers to perceive (e.g. increased stress hormone levels in nestlings under artificial illumination [66]). Each of these elements of habitat selection-attraction, deterrence, and perception of habitat quality - involves a series of cognitive processes that cannot be directly observed as outcome variables but may be inferred based on changes to movement or distributions. Providing the evidence for these inferences drives the primary question in our systematic map: How does artificial light affect bird movement and distribution?

Large bodies of research address two aspects of how artificial light affects bird movement and distribution: collision mortality of nocturnal migrants (e.g. [31, 72, 73]) and bird deterrence from zones of human-wildlife conflict (e.g. [74-76]). More recent studies use bird distributions to estimate the ecological impacts of ALAN on birds (e.g. [77, 78]). These literature bodies are typically synthesized separately (e.g. [28, 79]), despite shared emphases on bird movement and distribution. Evidence from each body of research is directly relevant to the others. For example, light-based deterrents that reduce the number of birds in zones of human-wildlife conflict could also reduce collision mortality of nocturnal migrants. Through our extensive literature search, we found and included in our map additional bodies of research that studied bird movement and distribution in response to artificial light. We sought to combine all the available evidence from multiple literature bodies to increase both the quantity of evidence available to inform review or management decisions and the diversity of species and light characteristics for which evidence is available.

\section{Stakeholder engagement}

Informal conversations with stakeholders from industries, government agencies, and non-profit organizations confirmed that this topic is relevant in many fields. We developed a questionnaire for stakeholders to inform our search strategy, identify secondary questions of particular importance, support map interpretation, and guide subsequent systematic reviews and primary research (Additional file 1 in our protocol [80]). Ten stakeholders responded to our questionnaire, helping us to expand our literature search and identify secondary questions of particular importance. Stakeholders included two academic researchers, four researchers or managers at government organizations, two non-profit leaders, and two industry stakeholders. The diversity of stakeholders was 
reflected in the broad range of topics identified as important, including the efficacy of deterrents, the contribution of ALAN to bird-building collisions, and the ecological impacts of artificial light in bird breeding and foraging habitat. All stakeholders were professional contacts of the authors, or were referred to us by other stakeholders who completed the survey, resulting in geographic bias, with nine of the ten stakeholders based in North America. We reached out to five additional stakeholders who did not respond. All stakeholders surveyed indicated that they were interested in how birds' behavioural responses to light are affected by light characteristics (e.g. wavelength, intensity, and direction). Future systematic reviews should engage in further stakeholder engagement to determine the level of interest in the evidence clusters we have identified.

\section{Objectives of the review}

Our primary question is:

\section{How does artificial light affect bird movement and distribution?}

We evaluated study relevance to our primary question according to the PI/ECO framework [81], characterizing the population, intervention or exposure, comparator (when available), and outcome for each study. We defined the PE/ICO elements as:

(P) Population: All bird species

(E or I) Exposure or Intervention: Anthropogenic light sources (including anthropogenic flames and electric light). We include both interventions (lights that are used to deliberately alter bird behaviour) and exposures (lights that are in place for a different reason).

(C) Comparator: Similar habitats, laboratory environments, structures, or landscapes that are unlit or lit with different types of light; same study site before or after intervention/exposure. Studies without a comparator were included in the map and the lack of comparator was recorded during metadata coding.

(O) Outcome: Bird movement through space, behaviours that precede movement through space (e.g. alert behaviour or orientation), or distributions (including presence/absence, use, abundance, or locations).

The first objective of our systematic map was to provide a database of evidence to answer questions about the effects of artificial light on bird movement and distributions. The results of the systematic map are contained in a database, which includes all eligible studies and metadata relating to the PE/ICO elements. The second objective was to provide an evidence base for four secondary questions that we identify below based on our expertise and consultation with stakeholders. Our third objective was to identify key evidence clusters (questions with sufficient evidence for review) and knowledge gaps (questions requiring more research) pertaining to each secondary question that are important from a conservation, management, or basic science perspective. Our secondary questions are:

1. What information is available documenting that birds in flight aggregate around and collide with artificial lights or structures with artificial lights?

2. What evidence exists on aggregation and mortality as a result of attraction and/or disorientation?

3. What information is available to describe the effect of light as a means of deterring or dispersing birds?

4. What evidence exists on continuous artificial illumination changing bird habitat selection for activities other than flight?

The primary question, the PE/ICO framework, and our secondary questions were published in our systematic map protocol [80]. We edited the language of our primary and secondary questions to reduce ambiguity and to allow us to identify relevant evidence based on population, exposures/interventions, comparators, and outcomes. We specified that our questions relating to aggregation/mortality and the causes of aggregation/ mortality focused on birds in flight because this is the context in which birds aggregate around lights and collide with illuminated structures. We specified that our question related to habitat selection excluded birds in flight because they have not yet settled in a particular location. We also narrowed the focus of this secondary question to continuous illumination because we focused on ecologically relevant light pollution, and flashing lights and lasers are rare in urban and suburban landscapes, outside of bird deterrence efforts. We incorporated the final secondary question from our protocol into all of our secondary questions, describing the range of weather, light, and population characteristics for which evidence is available.

\section{Methods}

This systematic map follows CEE guidelines [81] and ROSES reporting standards [82] (Additional file 1 ROSES form).

\section{Deviations from the protocol}

We altered our secondary questions to identify relevant studies based on PE/ICO elements. In order to increase the number of unique search results in our Google 
Scholar search, we used four search strings instead of the two written in our protocol. We used the program Publish or Perish [83] to save our Google Search results as . csv files. We changed our method for selecting reviews for bibliographic checking to identify reviews that focused on birds, artificial light, and one or more of our secondary questions. We added details to our eligibility criteria to promote transparency and consistency. We altered some of our metadata fields and codes to accommodate the diversity of studies included in the map. Records of these alterations and the final coding fields are listed in Additional file 11 (Data coding fields). Volunteers assisted with data coding. We further explain these changes to our protocol in the relevant sections below.

\section{Searching for articles}

We did not apply any document type restrictions to our search. All searches were conducted in English in Google Chrome using incognito mode. Although we did not include language restrictions in the database searches, we included only articles written in English. As described in our protocol [80], we compiled a list of 78 articles representative of the diverse studies that were eligible for inclusion in our map ("benchmark articles") and created a search strategy that found all of these articles.

\section{Database, citation indexing service, and website searches}

We used a search string consisting of population and exposure/intervention components of the primary question to search databases and citation indexing services. In the Web of Science Core Collection and in the Web of Science Zoological Record, we used the search string $\left(T S={ }^{*}\right.$ Bird" OR Avian OR Ave\$) AND (TS=Light* OR Laser* OR Strobe\$ OR Streetlight* OR Headlight\$ OR Spotlight* OR Lamp\$ OR Beacon\$ OR Beam\$ OR Flash* OR Flare\$ OR Flaring OR Reflector\$ OR Ceilometer\$). As outlined in our protocol [73], when searching for articles indexed without abstracts or keywords in the Web of Science Core Collection we expanded our population search string to include all English common family names (Additional file 2 WOS search strategy). We pre-screened the Web of Science search results to exclude articles that only used our exposure/intervention search terms in common irrelevant phrases (e.g. in light of, comes to light, or light-level geolocator), listed in Additional file 2. A complete explanation of this strategy and the macros used to implement it are provided in Additional files 2 and 3 (WOS search strategy and WOS search records). This strategy ensured that we found all benchmark articles indexed in Web of Science Core Collection or in Web of Science Zoological Record.

We also searched the following additional databases: Proquest Dissertations and Theses Global, Open Access
Theses and Dissertations, Conservation Evidence, Crop Protection Compendium, Tethys Knowledge Base, Artificial Light at Night (ALAN) Research Literature Database, NWRC Staff Publications, and Internet Center for Wildlife Damage Management, and Environmental Studies Program Information System (Additional file 4 Database search records). We modified our search string to fit the format of each database (Additional file 4). Finally, we searched the 18 websites that we identified in our protocol, using a combination of hand searching and built-in search functions on the website (Additional file 12; Supplementary tables and figures, Table S1). We used Google to search within the websites that were too large to feasibly manually check every page (Additional file 5 Website search records). All website searches were conducted on August 21, 2019, and were not updated due to time constraints. All Web of Science and specialized database searches were conducted in February or August 2019 and updated in August 2020 (Additional file 4 ).

\section{Web-based search engines}

We searched Google Scholar using Publish or Perish 6 [83] with the following search strings:

1. All of the words: Avian; Any of the words: Light Spotlight Streetlight Headlight Lamp Beacon Beam Ceilometer

2. All of the words: Avian; Any of the words: Laser Strobe Flash Flare Flaring Reflector

3. All of the words: Bird; Any of the words: Light Spotlight Streetlight Headlight Lamp Beacon Beam Ceilometer.

4. All of the words: Bird; Any of the words: Laser Strobe Flash Flare Flaring Reflector

We saved the first 1000 results from each search to.csv files (Additional file 6 Google Scholar search records). We combined all search results into a single Microsoft Excel spreadsheet, removed duplicates, and uploaded all search results to Rayyan QCRI for further screening (Additional file 6) [84]. Rayyan QCRI is a free web and mobile app for screening abstracts and titles for systematic maps and reviews. Using the program Publish or Perish caused a deviation from our protocol, but allowed us to retain a record of the Google Scholar search results and import them into Rayyan QCRI for screening. Using the four search strings, instead of the two written in our protocol, increased the number of unique search results.

\section{Assessing search comprehensiveness}

We chose one review for each secondary question for bibliographic checking. To select these reviews, we downloaded a list of all articles we had identified as reviews 
during screening in Rayyan QCRI and determined their relevance to each secondary question based on their titles. We then scored the articles based on the relevance and date of publication (details in Additional file 7 Bibliographic checking), giving higher priority to reviews that specifically focused on birds, artificial light, and one or more of our secondary questions. We randomly selected a review among the top scores for each secondary question and identified all eligible articles referenced in each review. When the first review of bird deterrence yielded only two relevant references, we randomly chose an additional review for this exercise. In total, we used five reviews to assess our search comprehensiveness, and we calculated the proportion of references for each review that were found with our systematic search.

Bibliographic checking confirmed that our search strategy found nearly all available evidence for most secondary questions. We found all four eligible references from the two reviews of deterrents $[79,85]$, all 17 eligible references from the orientation review [86], and eight out of 9 eligible references in the review relevant to habitat selection [87] (Additional file 7). Our search found 26 of the 44 eligible references in the review of aggregation/ mortality [28].

Finding all articles that documented birds aggregating or colliding around any structure with artificial lights (buildings, windows, towers, oil platforms, etc.) would require substantial additional search effort. Two of the 18 missed articles were theses and were only indexed in Open Access Theses and Dissertations. They did not include the terms "bird," "avian," or "Aves" in their titles (Additional file 7). However, the studies in these theses were included in the map because our search found peerreviewed articles reporting these studies. Eight of the 18 missed articles were not indexed in any of the databases we searched. Searching additional databases would not substantially improve the search comprehensiveness because none of these articles were indexed in Scopus and only one was indexed in JSTOR. The remaining eight articles were indexed in the Web of Science Zoological Record, but were not found because they did not include the term "light" or another exposure/intervention term in their topic fields. The shortest search string that could find all of these articles (TS $=($ "bird $*$ OR Avian OR Ave\$) AND TS $=$ (tower OR migration OR destruction)) returned 52,459 results, far more than we could feasibly screen.

\section{Article screening and study eligibility criteria}

We used Rayyan QCRI to screen search records from Web of Science, Google Scholar, and Proquest Dissertations and Theses Global. We first screened articles at the title and (if available) abstract level. Any articles excluded at the full text level are listed in Additional file 9 (Articles excluded at full text) with exclusion reasons. The remaining specialised databases did not provide an export to csv function, so they were screened in Microsoft Word if the text could be easily copied. Otherwise, they were screened on the website with the search results pages saved as PDF files. We initially screened using the information available on the webpage, recorded any articles that were potentially relevant, then made full-text eligibility decisions and recorded reasons for excluding any article (Additional file 9). CAA and $A B$ performed all screening, and neither screener authored any articles considered for inclusion/exclusion.

As we screened articles for study eligibility, we added details to the eligibility criteria published in the protocol to better define the scope of our map and promote consistency in the eligibility determinations (Table 1). We included further justifications for these eligibility criteria in Additional file 8 (Explanations of eligibility criteria). In accordance with our original criteria, we excluded specific exposures/interventions that did not involve an artificial light source, such as studies of reflected or filtered daylight. This necessarily excluded some studies of bird hazards (e.g. windows), deterrents (e.g. mylar, streamers, mirrors), and orientation (e.g. filters on natural daylight).

For interventions/exposures that are so broadly applied as to make mapping their use prohibitive, we required that they compared bird response under artificial light of different wavelengths or intensities. This criterion excluded laboratory studies using only broad-spectrum (i.e. white) light of a single intensity and a single photoperiod. We included laboratory studies that compared, within the same study, bird responses to light of varying intensities, different spectral compositions, or different photoperiods, providing that they also studied an eligible outcome.

In making eligibility decisions about outcomes, we included outcomes documenting movement through space or behaviours that directly precede movement (e.g. orientation or alert response). We excluded general locomotor activity (e.g. perch-hopping or overall activity levels). We excluded studies testing birds' ability to see specific wavelengths or using light as a visual cue to elicit a trained response. We excluded outcomes involving temporal changes in daily or annual movements (e.g. timing of entering/exiting nest boxes or migration), timing of daily activities (e.g. timing of feeding, sleeping, etc.), or circadian rhythms. We excluded studies of bird foraging behaviour that did not involve movement through space or changes to distribution (e.g. time spent foraging or changes to foraging strategies). We included incidental reports of nocturnal 
Table 1 Eligibility Criteria used to screen articles for inclusion in the systematic map

\begin{tabular}{|c|c|c|}
\hline & Eligibility criteria & Exclusion criteria \\
\hline Population & Any bird species, including domesticated species & \\
\hline Exposure/Intervention & $\begin{array}{l}\text { Anthropogenic light sources, including, but not limited to, point } \\
\text { sources of light, illuminated habitat, skyglow, and gas flares }\end{array}$ & $\begin{array}{l}\text { Reflected daylight (e.g. mylar tape reflectors, windows) } \\
\text { Filters on natural daylight }\end{array}$ \\
\hline Comparator & $\begin{array}{l}\text { Same site under different lighting conditions } \\
\text { Nearby site at the same time of day with similar habitat type, anthro- } \\
\text { pogenic activity, migration density (if applicable) and weather as the } \\
\text { treatment site } \\
\text { Documentation of aggregation, mass landing, mortality, foraging, or } \\
\text { other relevant outcomes near artificial light sources without com- } \\
\text { parator were included in the map and coded as "No Control" }\end{array}$ & $\begin{array}{l}\text { Laboratory experiments that use only one intensity or } \\
\text { spectral composition of light within the "light" or "dark" } \\
\text { portions of the light cycle } \\
\text { Laboratory experiments that use an artificial photo- } \\
\text { period but do not vary the photoperiod }\end{array}$ \\
\hline Outcome & $\begin{array}{l}\text { Bird density or presence/absence, including (but not limited to) radar } \\
\text { observations, visual counts and vocalization counts } \\
\text { Bird mortality } \\
\text { Resource consumption (such as crop damage or aquaculture preda- } \\
\text { tion) } \\
\text { Behavioural outcomes directly involving movement through space, } \\
\text { including (but not limited to) flight path, flight initiation, diving and } \\
\text { flushing } \\
\text { Behavioural response (flushing or immobilization) to spotlights used } \\
\text { for capturing birds } \\
\text { Behaviours known to precede movement: orientation, overt reaction } \\
\text { distance, alert response (e.g. moving head laterally or vertical, crouch- } \\
\text { ing) } \\
\text { Documentation of birds foraging under artificial lights } \\
\text { Habitat use metrics at foraging sites such as feeders (e.g. arrival/ } \\
\text { departure times, feeder visit rates) }\end{array}$ & $\begin{array}{l}\text { Responses that are not conventionally associated with } \\
\text { movement (e.g. hormonal responses) } \\
\text { Behavioural responses that do not include or directly } \\
\text { precede movement through space (e.g. preening, dust- } \\
\text { bathing, sleep behaviour, vocalizing) } \\
\text { General locomotor activity (e.g. perch-hopping or time } \\
\text { spent walking) } \\
\text { Temporal changes in daily or annual activity rhythms } \\
\text { (e.g. entering/leaving nest box, migration) } \\
\text { Changes to timing of activities in laboratory experi- } \\
\text { ments (e.g. feeding, drinking) } \\
\text { Total feed consumption in laboratory experiments } \\
\text { Trained responses (e.g. trained to perform a task when } \\
\text { a light is on) } \\
\text { Ability to see specific wavelengths (e.g. mate choice } \\
\text { or ability to distinguish objects under varying light } \\
\text { conditions) } \\
\text { Changes to timing in chick provisioning in field studies }\end{array}$ \\
\hline
\end{tabular}

foraging under artificial lights surrounded by dark habitat, while recording the comparator as None.

Two members of the map team (CAA and AB) conducted consistency checking by screening the first 200 articles at the title/abstract level in Rayyan QCRI (Additional file 10 Consistency check records), resulting in a Kappa score of 0.71. We clarified the eligibility criteria and repeated the consistency checking on an additional 200 articles, achieving 100\% agreement. CAA screened the remaining articles. In cases where study eligibility was unclear, CAA consulted with the mapping team and defined additional eligibility criteria. After CAA screened 7000 articles, AB and CAA repeated the consistency checking exercise. To correct for possible bias in the order in which the articles appeared on Rayyan, we screened a random selection of 200 articles from all Web of Science search results. We disagreed on the eligibility of only one article, resulting in a Kappa Score of 0.93. After further discussion, we included this article at the title/abstract stage, but ultimately excluded it at the full text level. In total, we conducted consistency checking on 400 (1.9\%) out of 21,150 articles screened on Rayyan QCRI at the Title/ Abstract level (including the updated search).
Two members of the map team (CAA and CCSC) conducted consistency checking on 20 randomly selected articles at the full text screening level (Additional file 10). After disagreeing on 7 articles, we discussed and refined the eligibility criteria until we agreed on all eligibility decisions. We employed an external screener, NL, who had not previously worked on the map to ensure our eligibility criteria were clear to a naive screener. After reading the eligibility criteria, NL made inclusion decisions for the 20 randomly selected articles. NL agreed with CAA and CCSC for 19 out of 20 articles, resulting in Kappa score of 0.77. After further discussion, NL agreed that the article in question should be excluded. In total, we performed consistency checking on 20/819 articles (2.4\%) screened at the full text level. CAA screened the remainder of the articles.

\section{Study validity assessment}

We did not conduct a study validity assessment because the diversity of context, discipline, and study design included in the scope of the map did not support consistent criteria for evaluating studies. To facilitate future assessments of study validity, we described the control and coded the type of control design (e.g. none, before/ 
after, control/impact, gradient-spatial, gradienttemporal), the data collection method (systematic or opportunistic), whether the study was observational or experimental (Additional file 11 Data coding fields).

\section{Data coding strategy}

We found a greater quantity of articles than we expected when writing our protocol, so we altered our data coding strategy in order to complete the systematic map. We hired a research assistant (NL) and engaged 25 undergraduate student volunteers to code metadata, each contributing on average $60 \mathrm{~h}$ (over $1500 \mathrm{~h}$ total). Undergraduate students were trained by CAA and typically worked in pairs to read articles and extract metadata while CAA supervised on live video calls. CAA proofread all data coding performed by volunteers, reading the abstracts and methods sections of the articles and confirming that metadata were correct. In articles without methods sections (e.g. lighthouse reports), CAA skimmed the articles to find relevant metadata. Any data coding performed solely by CAA or NL were similarly proofread by a volunteer. Any potential mistakes found by the proofreader were discussed by both readers until an agreement was reached. The questions posed by volunteers during data coding were answered and recorded in the coding instructions (Additional file 11 Data coding fields), helping to clarify our coding methods. This spreadsheet also lists and justifies all modifications made to the data coding fields outlined in our protocol. We coded any missing data as unavailable (UA). The large number of studies and metadata fields precluded us from obtaining missing data from the authors.

We conducted two data coding consistency checking exercises. In October 2018, three members of the mapping team $(\mathrm{AB}, \mathrm{CAA}$, and $\mathrm{SS})$ coded data from 10 articles selected randomly from all of the articles that were known to the authors at that time. Each article was coded by two readers. Minor inconsistencies that occurred in four of these articles were discussed and corrected. The only inconsistencies were between SS, the undergraduate volunteer, and one of the systematic map authors. In March 2020, CAA and NL performed an additional consistency checking exercise on 10 articles. Some inconsistencies occurred in the "Study Design" category, resulting in the addition of "Behavioural Assay-with control" and "Behavioural Assay-no control" to this field's codes. As part of training, volunteers demonstrated their skills by correctly coding data on two or three sample articles, but we did not complete a full consistency checking exercise with every volunteer. Instead, we used proofreading strategy described above.

Throughout the systematic map process, we identified diverse outcomes (i.e. response variables) that described bird behaviour, distribution, or community, adding outcome codes as necessary during data coding (Additional file 11). As the number of outcomes grew, we categorized them into subcategories to aid our synthesis. The subcategories for distribution outcomes are straightforward (e.g., bird locations, bird counts, mortality), while the behaviour subcategories require more explanation. Any description of flight path (e.g. velocity, altitude, direction) was included in the subcategory titled flight path characteristics. In the subcategory titled approach or flee response, we included any outcomes showing bird movement towards or away from light when not in flight (e.g. on the ground or water). There are other logical subcategories for grouping the behaviour outcomes, and we invite users to find studies with outcomes relevant to their specific questions using the search form in our database.

Community outcomes were not originally included in our PE/ICO framework, but we added community as an outcome category because studies reporting community metrics are relevant to our primary question. They measure the distributions (e.g. presence/absence or relative abundance) of groups of birds (e.g. species or functional groups) and summarise these data using various indices (e.g. species richness or functional diversity). The way in which the distribution data were interpreted determined if the study outcome category was coded as Distribution, Community, or both. If the study reported the presence/ absence or abundance of individual species, bird groups, or all birds as one group, we coded the outcome category as Distribution. If a study reported metrics that summarized the number or traits of species present, we coded the outcome category as Community. All but one study with a Community outcome also reported a Distribution outcome.

\section{Data mapping methods}

Our systematic map is a relational database in Microsoft Access (Additional file 13 Adams et al. 2021 Systematic Map) and in Microsoft Excel (Additional file 15: Adams et al 2021 Systematic Map Excel).The search function in the Access version allows the database to be filtered by any of the coded fields, returning a list of studies that meet the criteria chosen in the search form. In our Review Findings, we examine each metadata category (Context, Population,Exposure/ Intervention, Comparator, and Outcome) and describe the quantity of evidence for particular populations, exposures/interventions, and outcomes. For each of our secondary questions, we identified the available evidence based on PE/ICO filters that are relevant to these questions, as we have framed them (Table 2). Users of the database may refine these criteria to meet 
Table 2 Filters applied for each secondary question and quantity of evidence identified

\begin{tabular}{|c|c|c|c|c|c|}
\hline & \multirow{2}{*}{$\begin{array}{l}\text { Aggregation/ } \\
\text { mortality }\end{array}$} & \multicolumn{2}{|c|}{ Causes of aggregation } & \multirow[t]{2}{*}{ Deterrence } & \multirow[t]{2}{*}{ Habitat selection } \\
\hline & & Orientation & Attraction & & \\
\hline Population Filters & $\begin{array}{l}\text { Bird activity } \\
\text { during interven- } \\
\text { tion/expo- } \\
\text { sure= flying }\end{array}$ & & $\begin{array}{l}\text { Bird activity during } \\
\text { intervention/expo- } \\
\text { sure= flying }\end{array}$ & & $\begin{array}{l}\text { Bird activity during inter- } \\
\text { vention/exposure } \neq \text { flying }\end{array}$ \\
\hline $\begin{array}{l}\text { Exposure/ Intervention } \\
\text { Filters }\end{array}$ & & & & $\begin{array}{l}\text { Deterrent type = Laser, } \\
\text { Spotlight or Beam, Flash- } \\
\text { ing light, UV or near-UV } \\
\text { light, Line markers, or } \\
\text { Pyrotechnic }\end{array}$ & $\begin{array}{l}\text { Continuous/Flash- } \\
\text { ing = Continuous } \\
\text { AND } \\
\text { Deterrent Type } \neq \text { Laser }\end{array}$ \\
\hline Comparator Filters & & & & & $\begin{array}{l}\text { Control design } \neq \text { No } \\
\text { Control }\end{array}$ \\
\hline Outcome Filters & $\begin{array}{l}\text { Outcome } \\
\text { category= Dis- } \\
\text { tribution } \\
\text { OR } \\
\text { Outcome sub- } \\
\text { category= flight } \\
\text { path character- } \\
\text { istics }\end{array}$ & $\begin{array}{l}\text { Outcome subcat- } \\
\text { egory= Orientation } \\
\text { behaviour }\end{array}$ & $\begin{array}{l}\text { Outcome subcat- } \\
\text { egory= preferences } \\
\text { OR } \\
\text { Outcome= Change in } \\
\text { flight direction relative to } \\
\text { a light source, Capture of } \\
\text { birds in flight, Modeled } \\
\text { flight behaviour based on } \\
\text { observations of birds in } \\
\text { flight, Distance of flight } \\
\text { path from light source, or } \\
\text { flight altitude }\end{array}$ & $\begin{array}{l}\text { Outcome category= Dis- } \\
\text { tribution } \\
\text { OR } \\
\text { Outcome subcat- } \\
\text { egory= Approach or } \\
\text { Flee Response or Alert } \\
\text { behaviour } \\
\text { OR } \\
\text { Outcome = Change in } \\
\text { flight direction relative to } \\
\text { a light source, Capture of } \\
\text { birds in flight, Modeled } \\
\text { flight behaviour based on } \\
\text { observations of birds in } \\
\text { flight, Distance of flight } \\
\text { path from light source, or } \\
\text { flight altitude }\end{array}$ & $\begin{array}{l}\text { Outcome subcat- } \\
\text { egory= Preferences, Bird } \\
\text { counts, Bird Locations, or } \\
\text { Presence of foraging birds }\end{array}$ \\
\hline $\begin{array}{l}\text { Number of relevant } \\
\text { studies }\end{array}$ & 219 & 38 & 26 & 228 & 88 \\
\hline
\end{tabular}

their specific management or synthesis objectives. In our Review Findings, we describe evidence clusters and knowledge gaps for each secondary question.

Relevant evidence for our first question (aggregation/ mortality of flying birds) included studies that measure flight path characteristics, collision mortality, or the distribution of birds in flight. We filtered the database to include studies where the bird activity during intervention/exposure was coded as flying and the outcome measured was in the distribution category or in the flight path characteristics subcategory.

We also identified the potential evidence for two possible mechanisms of aggregation/mortality: attraction and disorientation. Evidence suggesting attraction included studies of flying birds that measured any of the following behaviour outcome subcategories: change in flight direction relative to a light source, capture of birds in flight, modeled flight behaviour based on observations of birds in flight, or flight altitude. We did not include outcomes related to bird distributions as evidence of attraction because a different relative abundance of birds in lit than unlit areas could be due to capture (failing to leave illuminated areas once they encounter them) rather than attraction (preferentially flying towards lights). Evidence suggesting disorientation included any study with outcomes in the subcategory of orientation behaviour.

To describe the evidence for our third secondary question, we identified the types of light used for the purpose of deterring birds. We included all studies using these types of light and reporting response variables that could demonstrate deterrence. We included studies of flying and non-flying birds because light is used to deter both flying birds (e.g. during aircraft flight) and non-flying birds (e.g. on airport runways and in agricultural fields). Any outcome in the category distribution could demonstrate deterrence if the light treatment reduces bird numbers or bird deaths. Behavioural outcomes in the subcategory approach or flee response or the subcategory alert behaviour could also demonstrate deterrence, as could any of the flight path characteristics outcomes that could suggest attraction (listed above).

Using our broad definition of habitat selection as a process rather than a specific study design, evidence relevant to our fourth secondary question includes several type outcomes documenting changes to bird presence, abundance, or other measures of distribution. The evidence 
included, but was not limited to, studies modeling relative selection probability by comparing the light levels at used to available locations [88]. We included any studies that measured one of the following outcome subcategories: preferences, bird counts, bird locations, or presence of foraging birds. We required studies to include a control treatment, control period, or light gradient in order to demonstrate whether the measured outcome was different in lit than unlit times or places. We only included studies involving continuous illumination, excluding lasers and flashing lights because they are not common in the built environment and are typically evaluated in the context of our third secondary question (deterrents). We excluded studies that only observed birds in flight because flying birds have not settled in a particular location that would demonstrate habitat selection.

\section{Review findings \\ Review descriptive statistics}

Our systematic search used multiple steps (Fig. 1), ultimately resulting in the manual screening of 26,208 records to return 469 eligible articles (Table 3). The majority of articles (351) came from the comprehensive searches of Web of Science Core Collection and Web of Science Zoological Record (Table 3), for which we applied several pre-screening processes (Additional file 12 Supplementary tables and figures: Figure S1). Specialized databases provided 61 unique articles, with 23 found in the Artificial Light at Night (ALAN) Literature Database, 14 found in Open Access Theses and Dissertations, and 9 found in the Internet Center for Wildlife Damage Management. An additional 10 articles were found on websites and 19 were found incidentally (e.g. recommended by stakeholders or found when searching for the full text of a different article).

We found documentation of bird response to artificial light dating back to 1880 and the number of studies has increased markedly since 1990 (Fig. 2a). Most studies were conducted in the Northwestern quadrant of the globe (290), and many were also conducted in the Northeastern (129) and Southeastern quadrants (53) (Additional file 12: Table S2). We found only 18 studies from the Southwestern quadrant. There were 127 studies involving light that was only turned on at night, 33 during the day only, and 10 at sunset or dusk only (Additional file 12: Table S3). Many other studies applied the light treatment at multiple times of day or did not specify when the lights were turned on.

Our interdisciplinary search found studies from many economic sectors with diverse study purposes. The most common sectors were transportation (126 studies) and urban/suburban/rural developments (123) (Table 4). Few studies came from the mineral mining or waste management sectors, despite the need to reduce bird numbers at toxic tailings ponds and landfills [89, 90]. Many studies were conducted to document and/or reduce bird aggregations or mortality (169 studies), while deterring birds (88), monitoring or describing bird migration (35), and documenting birds foraging under artificial light (47) were also common study purposes (Table 5).

Additional information about the number of articles found during each stage of the Web of Sciences searches is provided in Additional file 12: Figure S1.

\section{Mapping the quantity of evidence for the primary question} We fulfilled the first objective of our map by providing a database of all available evidence for our primary question regarding the effects of artificial light on bird movement and distribution. In this database (Additional files $13,14,15)$, we brought together literature from many research bodies that study relevant populations (birds), interventions/exposures (artificial light), and outcomes (movement and distribution). Others can easily search and filter this database with metadata related to PE/ICO elements to find evidence for specific management questions and future systematic reviews. The metadata and narrative descriptions provided for each study allow the user to quickly evaluate the relevance of each study to their question.

We provide three versions of the systematic map database. The Microsoft Access database (Additional file 13) can be opened and edited using Access, and the Microsoft Access ACCDE Database (Additional file 14) can be opened using the freely available program Microsoft Access Runtime [91]. Both versions of the database can be browsed using the VIEW form or searched using the SEARCH form, which produces a list of studies that meet metadata criteria specified by the user. We also provide a Microsoft Excel version of this database (Additional file 15), but we recommend using the Access version if possible to more easily search for studies and view the metadata fields.

\section{Population}

Broad reviews could target the most well-studied taxonomic orders. Of the 40 avian orders in IOC World Birds List [92], 26 were included in the map at least once (Table 6). Passeriformes (perching birds) was by far the best studied order, followed by Charadriiformes (shorebirds and relatives) and Procellariiformes (petrels, shearwaters, and albatrosses). Migrating birds were recorded in 188 studies, with an additional 29 studying fledgling seabirds migrating to sea and 22 studying a mix of migrating and non-migrating birds (Additional file 12: Table S4). Most studies (377/490) involved wild species 


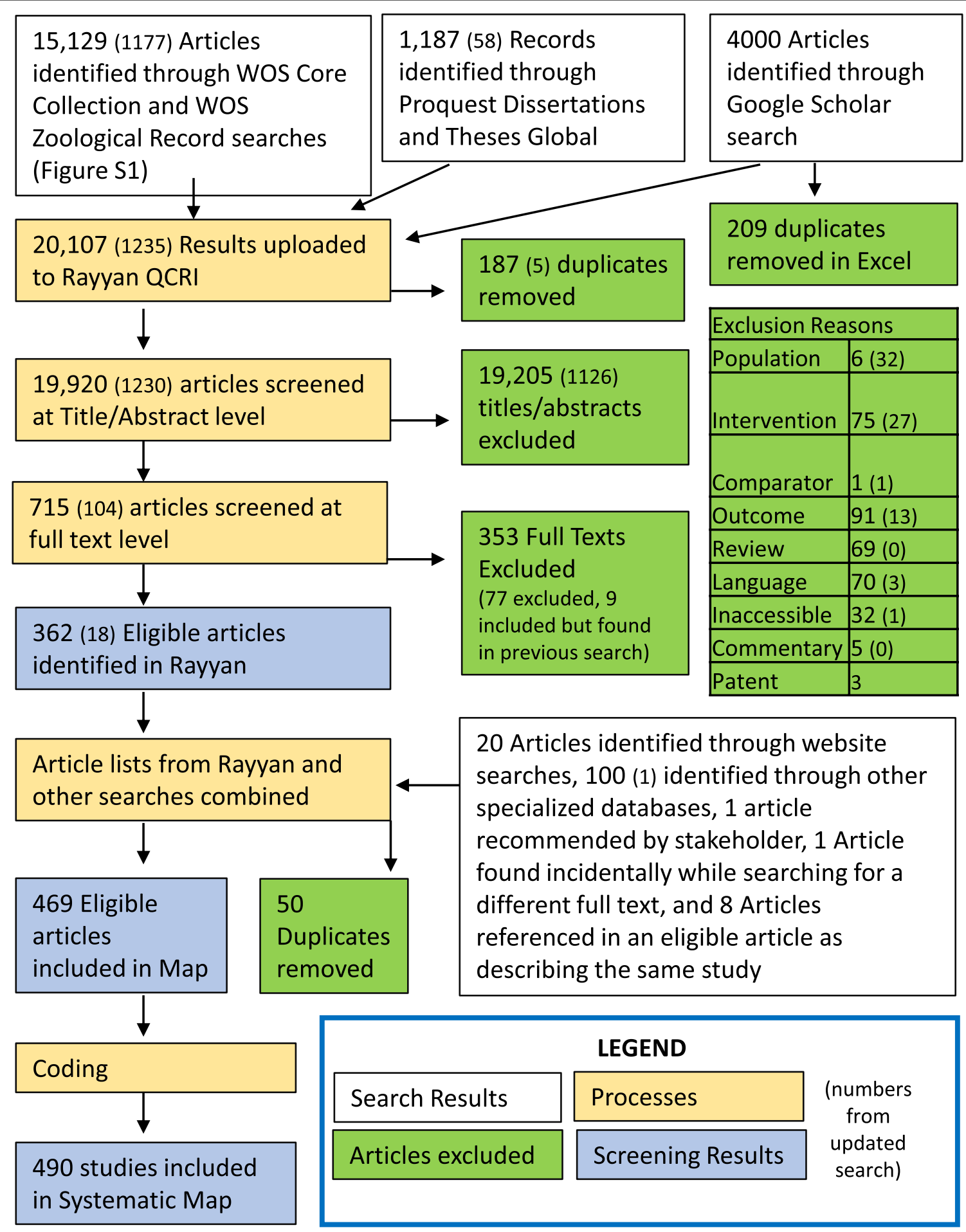

Fig. 1 Schematic of mapping stages including the search, screening, and coding stages

in the wild, while 81 studied wild species in captivity and 32 studied domesticated species (Additional file 12: Table S5).
Knowledge gaps may limit the application of these reviews for particular populations. Fourteen avian orders were never studied (Additional file 12: Table S6). Three of these orders are endemic to South America (e.g. 
Table 3 Number of articles found in each search step

\begin{tabular}{|c|c|c|c|}
\hline Source type & Captured articles & Eligible articles & Unique articles \\
\hline Web of Science (Core Collection) & $11,331(1,028)^{*}$ & $136(14)$ & $136(14)$ \\
\hline Web of Science Zoological Record & $6,353(417)^{* \dagger}$ & $205(9)$ & $199(2)$ \\
\hline Google Scholar & $3,791^{*}$ & 22 & 19 \\
\hline Proquest Dissertations at Theses Global & $1,187(58)$ & $8(1)$ & $8(1)$ \\
\hline Specialized Databases & $4,734(132)$ & $100(1)$ & $60(1)$ \\
\hline Organizational Websites & & 17 & 10 \\
\hline \multirow[t]{2}{*}{ Found Incidentally } & & 19 & 19 \\
\hline & Total: $29,031^{\dagger}$ & & Total: 469 \\
\hline
\end{tabular}

* These represent the number of articles captured after pre-screening with Microsoft Excel Macros (Additional files 2 and 3 ) and removal of duplicates (Additional file 12: Figure S1)

${ }^{\dagger}$ This is the number of articles captured from the Web of Science Zoological Record before removing articles also found in the Web of Science Core Collection (2555 such articles were removed in the original search, 149 in the updated search). After removing these duplicates, 26,208 articles were screened (21,342 in Rayyan, 4866 on websites or in downloaded documents).

Tinamiformes, Otidiformes, and Musophagiformes). More information on these orders may be available in Spanish or Portuguese, but our map only includes articles written in English. With only 18 studies conducted in the southwest quadrant of the globe (Additional file 12: Table S2), there is limited knowledge of how neotropical migrants respond to light during the non-breeding season, an important and understudied part of their life cycle [93] that can be key to increasing our understanding of bird collisions with buildings.

\section{Exposure/intervention}

There may be enough evidence to compare interventions/exposures that used well-studied wavelengths and flashing patterns and combinations of these two variables. The majority of studies (272/490) of continuous light were white or presumed white (described by study authors as white or presumed by the mapping team to be white based on context) (Fig. 3; Table 7). Continuous light was well-studied for other wavelengths visible to birds, including red (61), green (55), and blue (29). Only white and red were studied in more than 10 studies using flashing lights, while green, blue/green, and blue had 6, 6, and 8 studies, respectively. Rotating lights (a continuous light whose restricted visual angle rotates on a horizontal plane) were only included in 19 studies, but were likely also present in many of the 36 studies at lighthouses that were coded as UA (unavailable) because the text did not specify that the light rotated.

When identifying which light characteristics are important to study, it is important to consider the avian visual system. Orange, yellow, and UV or violet wavelengths were rarely studied (Table 7). UV and violet wavelengths are particularly important to consider for birds because some (but not all) bird species have a cone photoreceptor with peak sensitivity in the UV portion of the spectrum [86]. These wavelengths were only studied 13 times, and flashing UV or violet light was only studied three times. Different lamp types have distinct spectral compositions and flicker rates [94]. Birds have different spectral sensitivity and temporal visual resolution than humans $[95,96]$, so lamp types that all appear white to humans can appear distinct to birds [94, 95]. Only two studies using continuous light reported the flicker rate [97, 98]. Among the studies reporting lamp type, there were many using LED, Incandescent, Halogen, or Fluorescent bulbs for white lights (Table 8), providing an opportunity for review. A review comparing these lamp types could consider only white lights to avoid confounding lamp type with wavelength. Other lamp types that are commonly used for outdoor lighting were rarely recorded in our database, including Metal Halide, Mercury-Vapor, and Sodium Vapor [99], but were likely common in the 283 studies that did not report lamp type.

For wavelengths that were used in fewer than 5 studies (green/yellow, yellow/orange, and indigo), we recategorized them as the longer wavelength (yellow, orange, and blue) for this table and for all subsequent tables and figures. Wavelengths described by the article authors as turquoise were counted as blue/green.

\section{Outcome}

The fifty-five distinct outcome variables identified in our map demonstrate the diverse ways in which artificial light can change bird movement through space and distribution, with variety far beyond what we anticipated in our own systematic map protocol. We provide the first organized view of this literature, grouping outcomes into three categories (behaviour, distribution, and community) and eleven subcategories. This organization provides an easy way for map users to find evidence that documents particular outcomes or groups 
a) Total number of publications per year

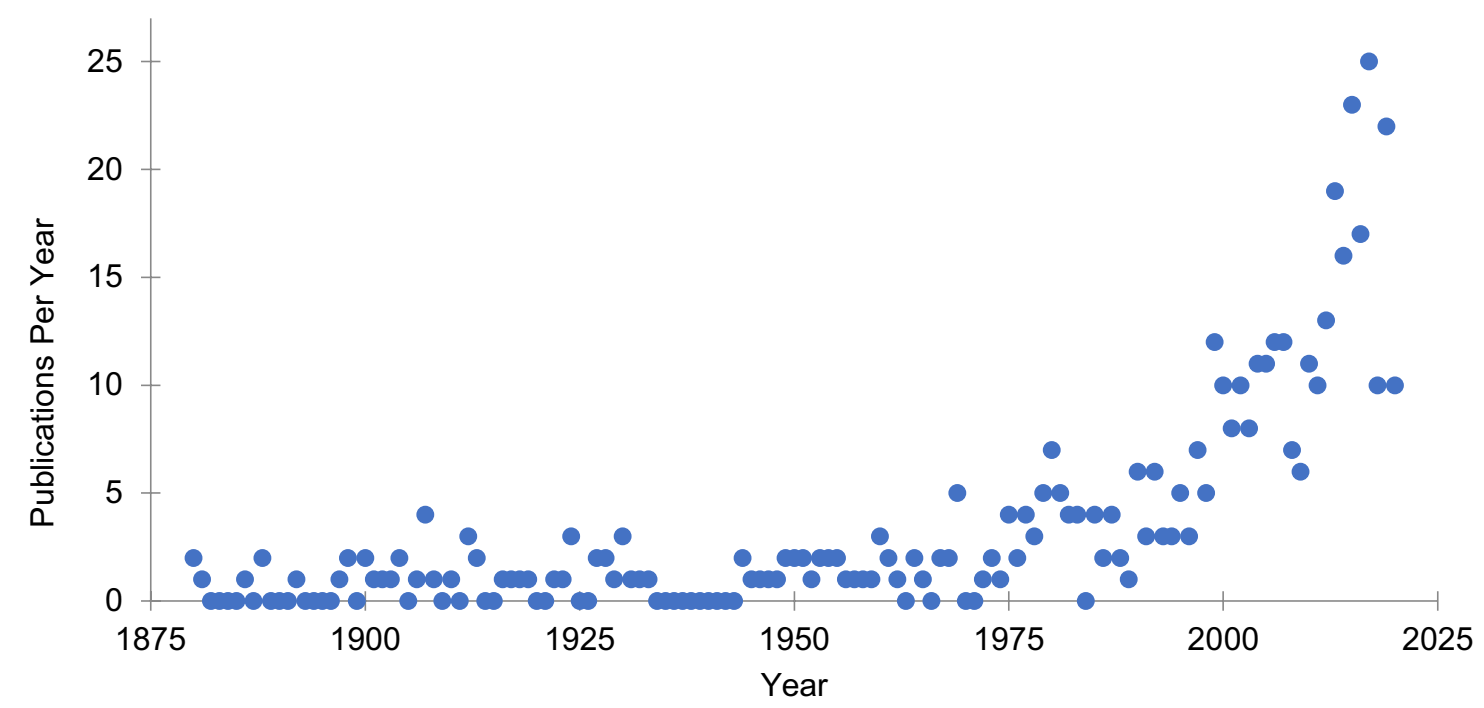

b) Number of publications per decade for the purpose of answering secondary questions

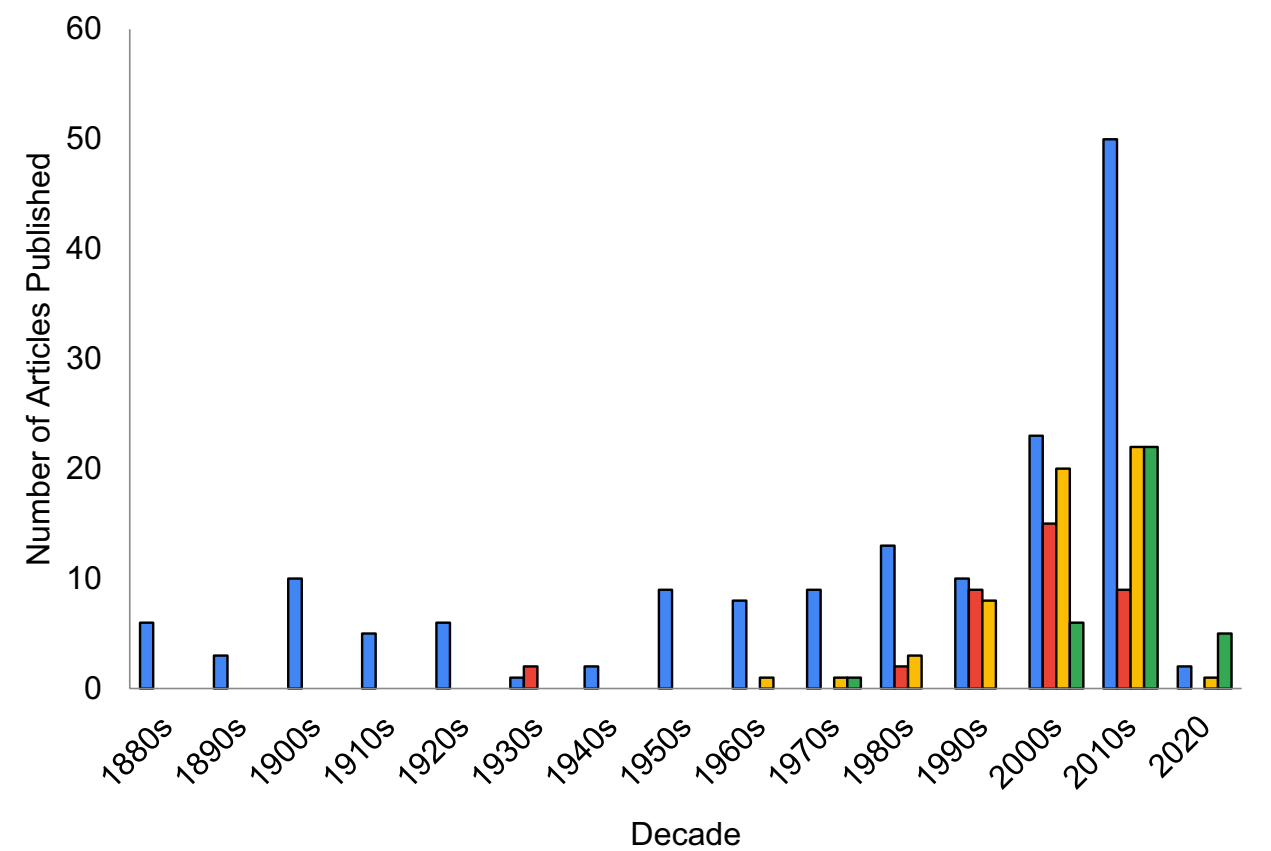

$\square$ Agg/.Mort

$\square$ Orient.

口Det.

口Hab.Sel.

Decade

Fig. 2 Publication count over time

of outcomes. Within the behavioural outcome category, there were four subcategories showing distinct types of movement or behaviours preceding movement and each subcategory includes several outcome variables (Table 9). Outcomes related to behaviour or distribution were each recorded in well over 200 studies, while only five studies recorded the impact of artificial light on bird communities. In the behaviour category, flight path characteristics, approach or flee response, and orientation were well studied, while studies documenting alert behaviour were relatively rare. All subcategories in the distribution category contained at least 25 studies. While outcomes related to behaviour and distribution were well-studied, there is not enough evidence to 
Table 4 The number of studies in each economic sector

\begin{tabular}{ll}
\hline Sector & $\begin{array}{l}\text { Number } \\
\text { of } \\
\text { studies }\end{array}$ \\
\hline Transportation & 126 \\
Urban/Suburban/Rural & 123 \\
Laboratory Behavioural Research & 56 \\
Wildlife Research & 54 \\
Food Production & 52 \\
Energy & 47 \\
Communication Towers & 16 \\
Recreation/Tourism & 10 \\
Human-wildlife Conflict-no sector specified & 10 \\
Natural Protected Area & 8 \\
Military & 5 \\
Zoos & 4 \\
Forestry & 2 \\
Port or Harbour & 2 \\
Waste Management & 2 \\
Mineral Mining & 1 \\
\hline
\end{tabular}

Table 5 The number of studies conducted for each study purpose

\begin{tabular}{ll}
\hline Purpose of study & $\begin{array}{l}\text { Number } \\
\text { of } \\
\text { studies }\end{array}$ \\
\hline Document and/or reduce bird aggregations or mortality & 169 \\
Deter birds & 88 \\
Document birds foraging under artificial light at night & 47 \\
Test ability to orient under artificial light conditions & 40 \\
Study effects of artificial light on bird distribution or habitat & 40 \\
selection & 38 \\
Capture or count birds for wildlife research & 36 \\
Improve welfare or production of captive birds & 35 \\
Monitor or describe bird migration & 7 \\
Basic behavioural research & 4 \\
Study effects of artificial light on bird community & 3 \\
Document occurrence of a rare species & 2 \\
Study weight loss during nocturnal migration & 2 \\
Document unusual bird behaviours & \\
\hline
\end{tabular}

inform conservation initiatives targeting avian community outcomes, such as species richness or functional diversity.

\section{Mapping the quantity of evidence relevant to each secondary question}

Our second objective was to provide an evidence base for four secondary questions with conservation and management importance, identifying relevant evidence based on objective $\mathrm{PE} / \mathrm{ICO}$ criteria rather than the authors' purpose for conducting the study (Table 2). Evidence for each question can be found using the Secondary Questions tab on the SEARCH form in our Access database (Additional files 13 and 14).

This interdisciplinary approach expanded the quantity and diversity of evidence for each question by including studies that were conducted for many different purposes. For example, evidence relevant to our secondary question about habitat selection came from 30 studies whose purpose was to improve welfare or production of captive birds and five studies conducted to deter birds (Additional file 12: Table S7). Evidence relevant to our secondary question about deterrence included 84 studies whose purpose was to document and/or reduce bird aggregations or mortality and 34 studies that captured or counted birds for wildlife research (Additional file 12: Table S7). By considering all studies with relevant populations, exposures/interventions, and outcomesrather than only considering studies that describe bird responses as attraction, deterrence, or habitat selectionreviews can draw on a broader evidence base with a greater diversity of bird species and light characteristics.

All four of our secondary questions received considerable study constituting evidence clusters, but aggregation/ mortality, deterrence, and habitat selection were studied more than disorientation and attraction (Table 2). Below, we describe the evidence clusters and knowledge gaps within each secondary questions and explain their importance from perspectives of conservation, management, or basic science.

\section{What information is available documenting that birds in flight aggregate around and collide with artificial lights or structures with artificial lights?}

Hundreds of papers documented bird aggregation or collision mortality in association with artificial light. There may be sufficient literature for a review to identify weather or lunar conditions or light characteristics that are associated with high probability or intensity of aggregation and mortality events, helping to target light reduction efforts on nights with those conditions. Such an analysis is likely possible because 75 studies made observations under multiple cloud cover/visibility conditions (Table 10) and 36 studies observed bird aggregation/mortality under multiple lunar phases (Table 11). The lunar phases in other studies could be calculated based on locations and dates. A more accurate representation of lunar illumination could include illuminated fraction and elevation, in addition to atmospheric conditions [100]. 
Table 6 Orders included in studies relevant to the primary question and each secondary question

\begin{tabular}{|c|c|c|c|c|c|c|c|}
\hline Order & Description & $\begin{array}{l}\text { Primary } \\
\text { question }\end{array}$ & $\begin{array}{l}\text { Aggregation/ } \\
\text { mortality }\end{array}$ & Disorientation & Attraction & Deterrence & $\begin{array}{l}\text { Habitat } \\
\text { selection }\end{array}$ \\
\hline Passeriformes & Perching birds & 258 & 141 & 33 & 14 & 118 & 36 \\
\hline Charadriiformes & Shorebirds and relatives & 87 & 50 & 0 & 6 & 56 & 9 \\
\hline Procellariiformes & Tube-nosed seabirds & 63 & 54 & 0 & 3 & 22 & 6 \\
\hline Galliformes & Landfowl & 44 & 7 & 2 & 0 & 18 & 22 \\
\hline Anseriformes & Waterfowl & 43 & 14 & 0 & 3 & 37 & 4 \\
\hline Gruiformes & $\begin{array}{l}\text { Diverse terrestrial and marshbirds, including } \\
\text { rails, cranes, and others }\end{array}$ & 27 & 18 & 0 & 1 & 21 & 0 \\
\hline Pelecaniformes & Ibis, herons, pelicans, and others & 23 & 11 & 0 & 0 & 11 & 2 \\
\hline Columbiformes & Pigeons & 18 & 6 & 3 & 0 & 6 & 3 \\
\hline Falconiformes & Falcons & 16 & 7 & 0 & 1 & 5 & 3 \\
\hline Suliformes & $\begin{array}{l}\text { Frigatebirds, cormorants, anhingas, and } \\
\text { boobies }\end{array}$ & 14 & 7 & 0 & 3 & 10 & 1 \\
\hline Caprimulgiformes & Nightjars & 14 & 5 & 0 & 0 & 2 & 4 \\
\hline Accipitriformes & Raptors including new world vultures & 14 & 7 & 0 & 3 & 6 & 3 \\
\hline Strigiformes & Owls & 12 & 5 & 0 & 0 & 3 & 5 \\
\hline Podicipediformes & Grebes & 9 & 6 & 0 & 0 & 6 & 0 \\
\hline Sphenisciformes & Penguins & 8 & 1 & 0 & 0 & 2 & 7 \\
\hline Coraciiformes & $\begin{array}{l}\text { Kingfishers and allies: rollers, todies, motmots, } \\
\text { bee-eaters }\end{array}$ & 6 & 3 & 0 & 0 & 1 & 1 \\
\hline Apodiformes & $\begin{array}{l}\text { Owlet-nightjars, treeswifts, swifts, humming- } \\
\text { birds }\end{array}$ & 5 & 0 & 0 & 0 & 0 & 1 \\
\hline Cuculiformes & Cuckoos & 4 & 3 & 0 & 0 & 1 & 1 \\
\hline Gaviiformes & Loons & 3 & 1 & 0 & 0 & 3 & 0 \\
\hline Ciconiiformes & Storks & 3 & 1 & 0 & 1 & 1 & 0 \\
\hline Piciformes & Woodpeckers and allies & 2 & 1 & 0 & 0 & 0 & 1 \\
\hline Apterygiformes & Kiwis & 2 & 0 & 0 & 0 & 0 & 2 \\
\hline Pterocliformes & Sandgrouse & 2 & 0 & 0 & 0 & 1 & 1 \\
\hline Psittaciformes & Parrots & 2 & 0 & 0 & 0 & 0 & 2 \\
\hline Rheiformes & Rheas & 1 & 0 & 0 & 0 & 1 & 0 \\
\hline Bucerotiformes & Hornbills, hoopoes, wood hoopoes & 1 & 1 & 0 & 0 & 0 & 0 \\
\hline
\end{tabular}

Order descriptions based on the IOC World Bird List [92]

A review of the evidence in this map could support or amend current recommendations for flashing instead of steady-burning lights (e.g. US Federal Aviation Administration [101] and Bureau of Ocean Energy Management [102]) and add details to these recommendations by determining how flashing rate influences bird response. International standards require that communications towers and other obstructions install combinations of red or white and flashing or continuous lights [103], and these towers are a significant source of mortality for birds [32]. Twenty-five studies included both red and white lights within the same study, and 25 included both continuous and flashing or rotating lights, providing an opportunity to compare the effects of these wavelengths or flashing patterns on bird aggregations and mortality when other conditions were held relatively constant.

There is insufficient evidence to compare the effects of different types of white light and few studies on wavelengths other than red. Most studies in this evidence base do not describe the spectral compositions of white light, and only 33 describe the type of light bulb used. In circumstances that require steady-burning white light (e.g. office towers, illuminated monuments, and airfield runway lighting), there may be an opportunity to change the spectral properties of white lights to reduce bird aggregation and mortality. Light of wavelengths other than red should also be studied because international standards require yellow and blue lights for mobile obstacles [103] and many other wavelengths are used for decorative lighting on bridges and buildings. In the evidence base for this secondary question, the green and blue each have only 7 studies each, while yellow and UV each have even fewer (Fig. 3).

There is also a knowledge gap concerning the relative danger of structures with flashing lights compared to unlit structures. Studies in the deterrent literature used 
a) Number of studies testing broad-spectrum white light

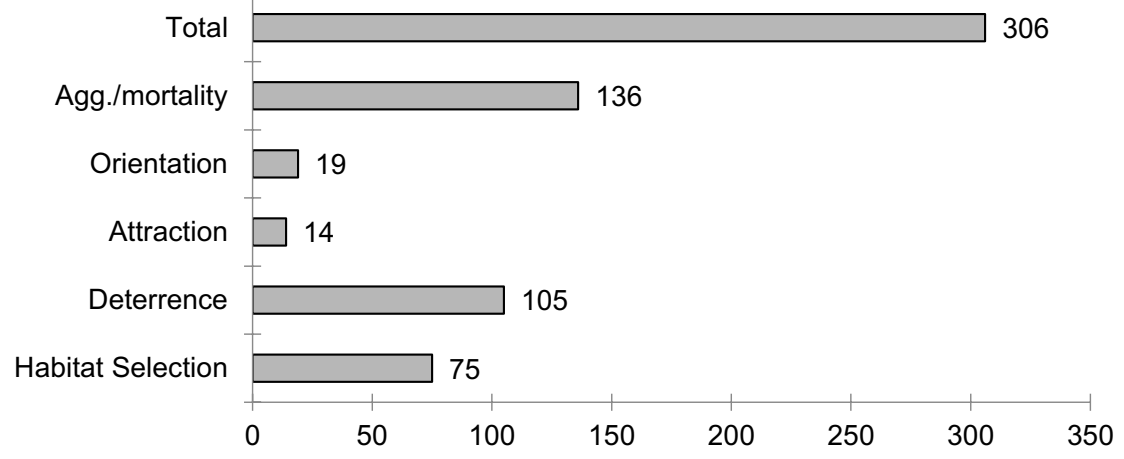

b) Number of studies testing each wavelength

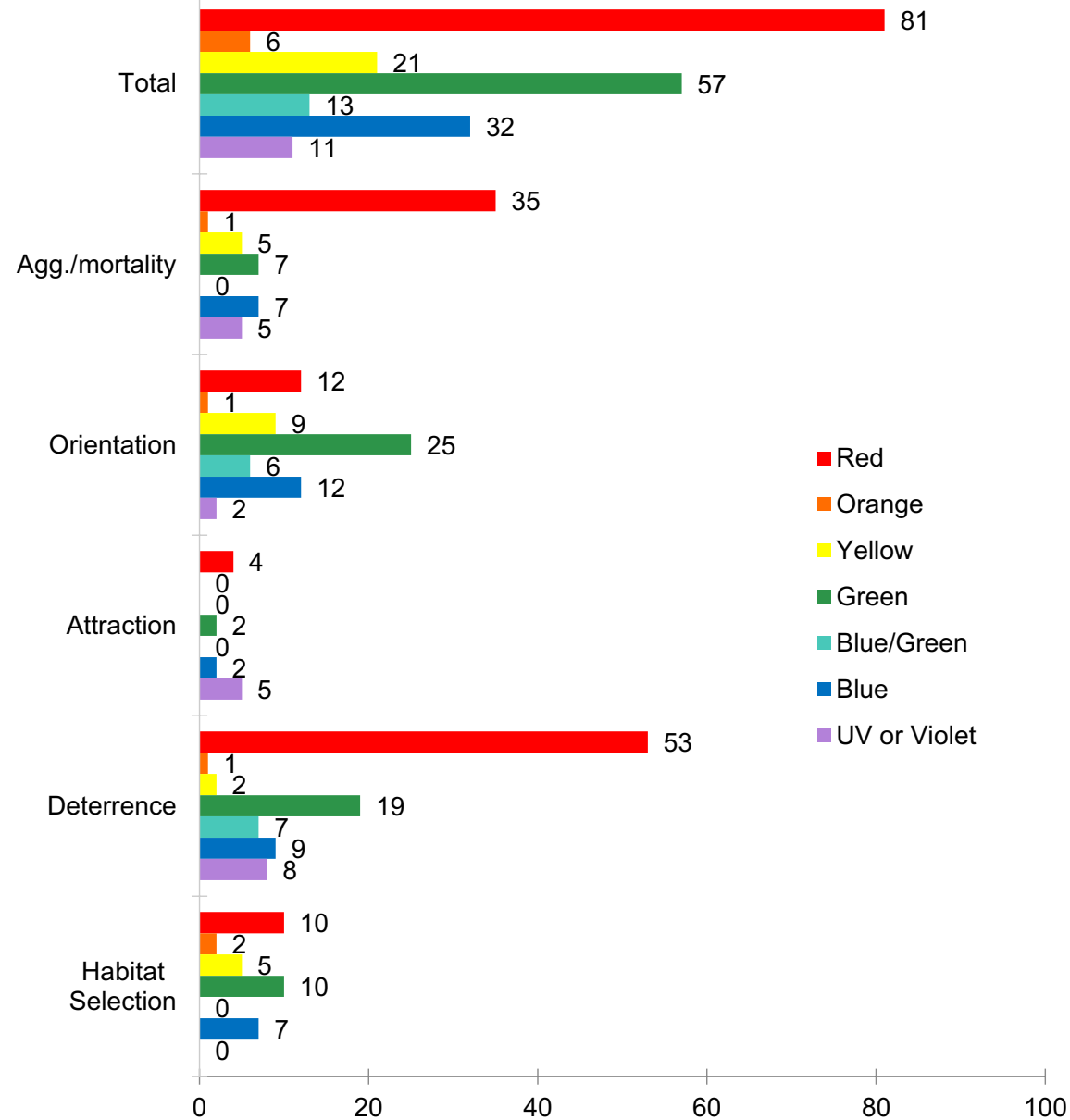

Fig. 3 Number of studies testing each wavelength of light in the evidence base and for each secondary question 
Table 7 Heat map showing the number of studies including interventions/exposures with each light wavelength, flashing pattern, and combinations of wavelengths and flashing patterns

\begin{tabular}{|l|r|r|r|r|r|r|}
\hline Wavelength & $\begin{array}{c}\text { Total } \\
\text { Number of } \\
\text { Studies }\end{array}$ & Continuous & Flashing & Rotating & $\begin{array}{c}\text { Simultaneous } \\
\text { flashing and } \\
\text { continuous } \\
\text { lights }\end{array}$ & Unavailable \\
\hline $\begin{array}{l}\text { White or } \\
\text { Presumed } \\
\text { White }\end{array}$ & 309 & 272 & 32 & 14 & 9 & 11 \\
\hline Infrared & 1 & 1 & 0 & 0 & 0 & 0 \\
\hline Red & 86 & 61 & 19 & 7 & 15 & 3 \\
\hline Orange & 6 & 6 & 1 & 0 & 0 & 0 \\
\hline Yellow & 22 & 20 & 2 & 0 & 0 & 0 \\
\hline Green & 59 & 55 & 6 & 0 & 0 & 0 \\
\hline Blue/Green & 13 & 13 & 6 & 0 & 0 & 0 \\
\hline Blue & 33 & 29 & 8 & 0 & 0 & 1 \\
\hline UV or Violet & 13 & 9 & 3 & 0 & 2 & 0 \\
\hline UV+ & 10 & 10 & 0 & 0 & 0 & 0 \\
\hline Flame & 10 & 10 & 0 & 0 & 0 & 0 \\
\hline Unavailable & 92 & 26 & 13 & 6 & 2 & 54 \\
\hline & TOTAL & & & & & \\
\hline
\end{tabular}

Blue shading represents the number of studies with each wavelength, from 1 (light blue) to 309 (dark blue). Yellow/red shading in columns three through seven represents the number of studies with each wavelength/flashing pattern combination, from 1 (pale yellow) to 61 (red). The TOTAL row shows the number of studies with each flashing pattern. The total is less than the sum of the columns because some studies included multiple wavelengths. UV + treatments added UV wavelengths to broad spectrum white light

Table 8 Number of studies including each lamp type

\begin{tabular}{|c|c|c|c|c|c|c|c|}
\hline \multirow[t]{2}{*}{ Lamp type } & \multirow[t]{2}{*}{ All } & \multirow{2}{*}{$\begin{array}{l}\text { White or } \\
\text { presumed white }\end{array}$} & \multirow{2}{*}{$\begin{array}{l}\text { Aggregation/ } \\
\text { mortality }\end{array}$} & \multicolumn{2}{|c|}{ Causes of aggregation/mortality } & \multirow[t]{2}{*}{ Deterrence } & \multirow{2}{*}{$\begin{array}{l}\text { Habitat } \\
\text { selection }\end{array}$} \\
\hline & & & & Attraction & Orientation & & \\
\hline Unavailable & 285 & 202 & 165 & 13 & 8 & 135 & 42 \\
\hline LED & 72 & 38 & 16 & 6 & 28 & 20 & 18 \\
\hline Laser & 45 & 0 & 0 & 0 & 0 & 45 & 1 \\
\hline Incandescent & 37 & 35 & 8 & 1 & 7 & 11 & 16 \\
\hline Halogen & 27 & 21 & 9 & 2 & 5 & 13 & 4 \\
\hline Fluorescent* & 21 & 20 & 4 & 0 & 1 & 2 & 13 \\
\hline Flame & 15 & 3 & 15 & 3 & 0 & 5 & 0 \\
\hline $\begin{array}{l}\text { Sodium Vapor } \\
\text { (Low or High Pressure)* }^{*}\end{array}$ & 11 & 10 & 7 & 0 & 0 & 1 & 2 \\
\hline Mercury-Vapor* & 9 & 6 & 6 & 0 & 0 & 5 & 0 \\
\hline Metal Halide* & 8 & 8 & 6 & 0 & 0 & 1 & 1 \\
\hline Xenon & 3 & 2 & 1 & 1 & 2 & 0 & 0 \\
\hline Electric Light (type UA) & 3 & 2 & 3 & 0 & 0 & 2 & 0 \\
\hline Neon & 1 & 1 & 0 & 0 & 1 & 0 & 0 \\
\hline Multi-metal Vapor & 1 & 1 & 0 & 0 & 0 & 0 & 1 \\
\hline Magnesium & 1 & 1 & 1 & 1 & 0 & 1 & 0 \\
\hline
\end{tabular}

Table shows the number of studies testing each lamp type in the whole evidence base, for all studies testing white light (or light presumed by the mapping team to be white), and in the evidence base for each secondary question

*Dominant lighting types for older streetlights and other outdoor lighting [82]

flashing lights or UV lights to attempt to prevent collisions by increasing the bird's ability to detect an object in its path (e.g. [104]), but there have been no studies comparing collision rates at tall structures with unlit control structures. Most tall structures require aviation safety lights, but new technology may permit unlit structures by using radar detection to turn on obstruction lighting only when an aircraft approaches [105]. While structures 
Table 9 Counts of Outcomes, Outcome subcategories, and Outcome Categories

\begin{tabular}{|c|c|c|c|}
\hline $\begin{array}{l}\text { Outcome category } \\
\text { (count) }\end{array}$ & Outcome subcategory (count) & Outcome & Study count \\
\hline \multirow{30}{*}{$\begin{array}{l}\text { Behaviour } \\
\text { (229) }\end{array}$} & \multirow[t]{3}{*}{ Alert behaviour (9) } & Other alert response & 6 \\
\hline & & Overt reaction distance & 2 \\
\hline & & Alert reaction time & 1 \\
\hline & \multirow{11}{*}{$\begin{array}{l}\text { Approach or flee response } \\
(67)\end{array}$} & Flying & 31 \\
\hline & & Capture of birds on the ground or water & 28 \\
\hline & & Flush (unspecified) & 23 \\
\hline & & Immobilization & 16 \\
\hline & & Diving & 12 \\
\hline & & Running or walking & 10 \\
\hline & & Unspecified & 8 \\
\hline & & Distance moved & 5 \\
\hline & & Other flee response & 3 \\
\hline & & Swimming & 3 \\
\hline & & Head movement & 2 \\
\hline & \multirow[t]{12}{*}{ Flight path characteristics (123) } & Circling, hovering, or other description of "attraction" & 77 \\
\hline & & Observations of bird collisions & 50 \\
\hline & & Altitude & 19 \\
\hline & & Direction & 13 \\
\hline & & Linear/non-linear flight path & 12 \\
\hline & & Velocity & 7 \\
\hline & & Change in flight direction relative to light source & 5 \\
\hline & & Capture of birds in flight & 4 \\
\hline & & Distance of flight path from light source & 2 \\
\hline & & Distance travelled & 2 \\
\hline & & $\begin{array}{l}\text { Modeled flight behaviour based on observations of bird locations or } \\
\text { distributions }\end{array}$ & 2 \\
\hline & & Other flight behaviour & 2 \\
\hline & \multirow{4}{*}{$\begin{array}{l}\text { Orientation behaviour } \\
\text { (40) }\end{array}$} & Ability to orient in migratory direction & 32 \\
\hline & & Ability to locate reward & 3 \\
\hline & & Other orientation behaviour & 3 \\
\hline & & Homing & 2 \\
\hline \multirow{6}{*}{$\begin{array}{l}\text { Community } \\
\text { (5) }\end{array}$} & \multirow{2}{*}{$\begin{array}{l}\text { Composition } \\
\text { (3) }\end{array}$} & Abundance of species grouped by type & 2 \\
\hline & & Stability & 1 \\
\hline & \multirow{4}{*}{$\begin{array}{l}\text { Diversity } \\
\text { (5) }\end{array}$} & Species richness & 5 \\
\hline & & Beta diversity & 1 \\
\hline & & Evolutionary distinctiveness & 1 \\
\hline & & Functional diversity & 1 \\
\hline
\end{tabular}


Table 9 (continued)

\begin{tabular}{|c|c|c|c|}
\hline $\begin{array}{l}\text { Outcome category } \\
\text { (count) }\end{array}$ & Outcome subcategory (count) & Outcome & Study count \\
\hline \multirow[t]{20}{*}{ Distribution (372) } & \multirow{2}{*}{$\begin{array}{l}\text { Bird counts } \\
(140)\end{array}$} & Abundance or density & 101 \\
\hline & & Presence/absence & 40 \\
\hline & \multirow[t]{6}{*}{ Bird locations (26) } & Locations of individual birds & 12 \\
\hline & & Locations of nests & 5 \\
\hline & & Locations of roosts or groups of birds & 3 \\
\hline & & Light exposure of tracked individuals & 3 \\
\hline & & Mean or median light exposure of a species & 2 \\
\hline & & Proximity of dead or injured birds to artificial light sources & 1 \\
\hline & \multirow{5}{*}{$\begin{array}{l}\text { Mortality } \\
(150)\end{array}$} & Counts or presence of dead or injured birds & 134 \\
\hline & & Locations of dead or injured birds & 10 \\
\hline & & Absence of bird deaths or injuries & 5 \\
\hline & & Seabird bycatch & 5 \\
\hline & & Type of mortality observation UA & 5 \\
\hline & \multirow{2}{*}{$\begin{array}{l}\text { Preferences } \\
\text { (61) }\end{array}$} & Free choice (time spent in each lighting option) & 46 \\
\hline & & Discrete choice (instantaneous choice among lighting options) & 15 \\
\hline & \multirow[t]{5}{*}{ Presence of foraging birds (56) } & Multiple individuals foraging under artificial lights & 38 \\
\hline & & One individual foraging under artificial lights & 12 \\
\hline & & Amount of food consumed & 4 \\
\hline & & Number of foraging events (individuals not distinguished) & 3 \\
\hline & & Arrival or departure time of foraging birds & 2 \\
\hline
\end{tabular}

Numbers in parentheses show the number of studies in each outcome category and outcome subcategory

without lighting may cause less attraction or disorientation, birds will not be alerted to the presence of dark structures in their flight path [106]. Before this new technology is adopted, it will be important to compare bird

Table 10 Cloud Cover/Nisibility in studies relevant to aggregation/mortality

\begin{tabular}{lc}
\hline Cloud cover/visibility & Study count \\
\hline Only clear days/nights & 6 \\
Only cloudy days/night & 29 \\
Varied-descriptive & 31 \\
Varied-events only & 17 \\
Varied-events only and descriptive & 2 \\
Varied-systematic & 25 \\
UA & 107 \\
\hline
\end{tabular}

Cloud Cover/Visibility was coded as Varied - systematic if it was recorded at regular intervals throughout the study period. It was coded as Varied - events only if it was only recorded during aggregation/mortality events and Varieddescriptive if authors described in general terms the conditions under which aggregation/mortality were most likely to occur mortality at dark structures to structures with various types of lights, particularly flashing or UV lights that are sometimes used as deterrents.

Table 11 Lunar phase in studies relevant to aggregation/ mortality

\begin{tabular}{lc}
\hline Lunar phase & Study count \\
\hline New & 3 \\
Full & 0 \\
In between & 1 \\
Varied-systematic & 26 \\
Varied-descriptive & 7 \\
Varied-events only & 2 \\
Varied-other & 1 \\
UA & 176
\end{tabular}

Lunar phase was coded as Varied_-systematic if it was recorded at regular intervals throughout the study period. It was coded as Varied - events only if it was only recorded during aggregation/mortality events and Varied-descriptive if authors described in general terms the conditions under which aggregation/ mortality were most likely to occur 


\section{What evidence exists on aggregation and mortality as a result of attraction and/or disorientation?}

While many authors assume that aggregations and mortality result from attraction, we only identified 26 studies with response variables that could suggest attraction (Table 2). By definition, these studies can also suggest deterrence if birds fly away from the light source. A review could describe whether birds show opposing responses depending on light characteristics (e.g. moving v. stationary, flashing v. continuous). Identifying where attraction occurs and the mechanism of attraction could help design lighting that is safer for birds. However, the relatively small number of relevant studies limits the potential for review, with only 14 studies including white light, 5 including UV/violet, and even fewer studies including red, green, or blue wavelengths (Fig. 3).

To conclusively demonstrate attraction to light, birds must be given a simultaneous choice between light treatments when all other conditions are identical (i.e., choice tests, [107]). Only one study used a controlled choice test on birds in flight [97] (Additional file 12: Table S8). Two studies modeled flight behaviour based on observations of bird locations or distributions [31,33], but did not directly observe their flight paths. Twenty-three studies recorded a change in altitude, flight direction relative to a light source, or capture of birds in flight, but did not offer simultaneous choice. Further choice tests could determine the light characteristics and ambient conditions under which attraction is most likely to occur, with relevance for both reducing attraction and more effectively deterring birds.

Birds flying around lights (e.g. circling, fluttering, hovering) may be disoriented, but only one orientation mechanism has been extensively tested. Thirty studies tested bird magnetoreception (Additional file 12: Table S9), and a review concluded that birds cannot perform magnetoreception in the complete absence of shortwavelength light from UV to green $(565 \mathrm{~nm})$ [49]. Some conservation biologists have speculated that the long wavelengths in broad-spectrum (i.e. white) light disorient birds by interfering with magnetoreception [73], but a review of laboratory research concluded that birds orient appropriately under broad-spectrum light that contains these wavelengths [49]. It is unclear whether birds can perform magnetoreception under all types of white light because only incandescent, halogen or xenon white lamps were tested in the twelve magnetoreception studies using white light (Additional file 12: Table S10). These lamps have broad emissions spectra, while other lamp types (e.g. metal halide and some LEDs) have distinct spectral peaks [99]. These spectral peaks may interfere with magnetoreception, as is the case for monochromatic and bichromatic light $[49,108]$. Two magnetoreception studies did not report the direction of the light, and the remaining 28 used overhead lights to illuminate Emlen funnels [109], mazes, or cages. These conditions differ from the built environment that birds experience on migration, where light intensity can vary across a bird's visual field (e.g. when flying in or above cities).

Loss of a single orientation mechanism may not disorient birds because the aviation orientation system includes redundancy [39]. Only eight studies tested how artificial light interferes with orientation mechanisms other than magnetoreception, including the disorienting effects of horizon glow (3), clock-shifts (3), and photoperiod changes (2) (Additional file 12: Table S9). None tested the impacts of skyglow or satellite clusters on the ability for birds to orient using their stellar compass. Together, conservation biologists and sensory ecologists should collaborate to design studies that fill these knowledge gaps and determine how the many types of artificial light common in the built environment interfere with some or all of birds' orientation mechanisms.

\section{What information describes the effect of light as a means of deterring or dispersing birds?}

Lasers formed the most promising evidence cluster for meta-analysis of studies specific to deterrent efficacy, while spotlights and flashing lights could be reviewed while including studies documenting bird aggregation, deterrence, and capture. A review might include red, green, and blue green lasers (studied 21, 10, and 7 times, respectively), while blue and violet were each studied only once (Additional file 12: Table S11). Forty of the 42 studies using lasers measured an approach or flee response, offering a common set of outcomes on which to base a meta-analysis. A meta-analysis of laser efficacy would be particularly useful because they are commonly sold as bird deterrents and were by far the most common type of light-based deterrent in our map (Table 12).

For flashing lights and spotlights, including evidence from studies with other purposes increased the size of the evidence base (Table 12). A review of bird response to flashing lights and spotlights would have applications for reducing aggregations, improving deterrents, and capturing wildlife. Spotlights were commonly documented in the aggregation/mortality literature, primarily at lighthouses, and in studies whose purpose was to capture birds for wildlife research (Table 12). When capturing birds, many authors recorded whether each species would typically flee or freeze in response to the spotlight. With the 21 taxonomic orders studied at least once in the spotlight studies, there may be an opportunity to investigate which species traits impact the efficacy of spotlights as deterrents or trapping tools. We did not record the characteristics of the spotlight used (e.g. light 
intensity, beam angle, predictability of movement) in this map, but a review could compare these characteristics to identify important predictors of bird responses. Deterrent efficacy may depend on ambient light levels, which change over the course of the day [27]. The evidence base may support a review of this topic, with 23 studies that applied the intervention/exposure at multiple times of day, 18 studies conducted only during the day, and 72 studies conducted only at night (Additional file 12: Table S12).

More research is needed on ultraviolet and violet light as a deterrent and the potential for light-based deterrents to reduce bird collisions or entanglement in wires and lines. UV or violet light was only tested in 8 studies in the deterrence evidence base (Fig. 3), and only once for lasers (Additional file 12: Table S11). Orange and yellow were included in fewer studies, UV/violet could be a particularly important knowledge gap because many bird species have a cone receptor with peak sensitivity in the UV or violet portions of the spectrum [96]. For example, a vision model indicated that light peaking in the UV/violet range (380-400 nm) would be most visible to Canada Geese (Branta canadensis), which are common targets of laser deterrent efforts, under a specific set of visual conditions (i.e., certain objects with specific types of illumination) [76]. In general, deterrent studies would benefit from more information about the spectral sensitivity of target species [53]. In addition to alerting birds to approaching vehicles or dispersing birds from zones of human-wildlife conflict, light-based deterrents were used in a few cases to increase awareness of stationary obstacles like power lines [104] and fishing lines [110112]. More research is needed to determine what types of lights are effective for warning birds about obstructions without inducing bird aggregations [106, 113]. Like attraction, the strongest evidence for deterrence comes from controlled choice tests, but these were rare. Eighteen studies, coming from only seven unique articles, conducted choice tests using light sources typically used as deterrents (lasers, UV/violet lights, flashing lights, or spotlights) (Additional file 12: Table S13). Choice experiments could greatly improve our understanding of the potential for different types of light to attract or deter birds and how the effects depend on species, light characteristics, and context of the intervention/exposure.

\section{What evidence exists on continuous artificial illumination changing bird habitat selection for activities other than flight?}

A review of the 88 studies for this secondary question would include 20 different bird orders, providing an opportunity to examine how life history or physiological traits influence selection for or avoidance of artificially illuminated environments. Examples of traits that could influence whether a species prefers or avoids artificially illuminated habitat patches include eye morphology [114], nocturnality [2], and foraging strategy [13]. Much of the continuous illumination experienced by birds in the wild comes from outdoor lighting like streetlights and illuminated signs, which are rapidly converting from older technology to LED [1]. To study whether LEDs have different effects on habitat selection than other types of white light, a review could compare the results of studies using fluorescent, incandescent, and LED lights (each studied at least 13 times, Table 8). However, such a review may be limited because LEDs can have varying spectral properties and the spectral power distribution of any light is rarely described. A few studies tested blue, green, and red light, providing further opportunity to review how birds respond to different parts of the visual spectrum (Fig. 3). Because study design varied widely across the evidence base, a review of the impacts of artificial light on bird habitat selection must incorporate multiple study designs to include sufficient evidence for meaningful inference. Seven studies measured relative abundance, while 16 modeled probability of selection

Table 12 Number of studies documenting bird response to each deterrent type

\begin{tabular}{lcc}
\hline Deterrent type & Number of studies using deterrent type to deter birds & $\begin{array}{l}\text { Total number of studies } \\
\text { documenting bird response to } \\
\text { deterrent type }\end{array}$ \\
\hline Laser & 46 & 46 \\
Flashing light & 23 & 68 \\
Spotlight & 11 & 158 \\
UV or near-UV light & 7 & 9 \\
Line markers & 4 & 4 \\
Pyrotechnic & 1 & 1 \\
\hline
\end{tabular}

The first column shows the number of studies using each deterrent type to intentionally deter birds. The second column shows the number of studies documenting bird responses to each deterrent type with outcomes that could demonstrate deterrence, regardless of the purpose for which the study was conducted 
or occupancy (Additional file 12: Table S14). Nineteen studies used other types of control/impact, before/after, or gradient designs. The 43 preference tests could form an evidence cluster, but 20 of these tested domestic poultry species, which may have little application to wildlife [115]. While incorporating diverse study designs expands the evidence base, it may limit the comparison of results across studies.

For habitat selection, knowledge gaps remain for nonpasserine species, many lamp types commonly used for outdoor lighting, and different spectral properties in LED lamps. Only Passeriformes (perching birds), Galliformes (landfowl), Procellariiformes (tube-nosed seabirds, including petrels, shearwaters, and albatrosses), and Charadriiformes (shorebirds and relatives) were studied more than five times, limiting the ability to predict how other taxonomic orders will respond to the global increase in ALAN (Table 6). As older street lamps are converted to LED, the choice to convert and the type of LED chosen will alter the night-time environment for decades to come, potentially changing both the sensory experience and prey availability for birds [14, 116-118], which may impact their habitat selection. LED lamps vary in their spectral properties and intensities even within comparable costs and energy efficiencies [116], so there may be opportunities to choose lights that are less likely to impact bird habitat selection while keeping costs low. We found that there is little evidence on the impacts of different types of white light on bird habitat selection, with very few studies using lamp types common for older streetlights and outdoor lighting (Table 8) and none reporting the spectral power distributions of white light.

Habitat selection is a particularly important domain for understanding the effects of artificial light on birds because it has widespread implications for both management and conservation. Changing or reducing artificial light can discourage birds from settling in areas where they pose a danger or nuisance to humans. For example, three studies in our map showed how artificial light levels can influence roost site selection for nuisance species in urban environments [119-121]. Another study changed the colour of runway lighting to reduce insect attraction, thereby reducing the number of birds on the runway [122]. From a conservation perspective, understanding the impacts of ALAN on habitat selection can help target light abatement efforts towards species that are most negatively impacted. As ALAN increases, species that avoid using illuminated areas will suffer reduced habitat availability regardless of whether habitat quality is affected. Species that select artificially illuminated habitat despite low reproductive success may face ecological traps and population declines [71]. The evidence in this map can identify loss in habitat availability and potential ecological traps, but does not include evidence of ALAN's effects on bird fitness other than collision mortality. Reviews or further research should also consider additional metrics (e.g. survival or reproduction rates) when evaluating the ecological impacts of artificial light on birds [57].

\section{Mapping the quality of evidence}

Further critical appraisal is necessary to determine the number of articles that could be included in systematic reviews. Requiring a comparator would disqualify approximately a third of the evidence in the map, and further critical appraisal may reduce the evidence base. To aid users in assessing how many studies are likely to pass critical appraisal and determining if there is sufficient evidence for a systematic review, we coded each study's control design in the database and wrote a description of the control treatment.

The majority of studies included a comparator, with light characteristics (e.g. presence/absence, intensity, wavelength, polarization, total illuminated area) varying across treatment periods or sites in 290 of 490 studies (Additional file 12: Table S15). The remaining 200 studies had no comparator but offer evidence of bird response to light if certain assumptions are accepted about bird behaviour or distribution in the absence of artificial light (Additional file 12: Table S15). For example, reports of birds aggregating around artificial light sources attribute the aggregations to the presence of light, assuming that aggregations did not occur in unilluminated areas. Fiftyone behavioural assays recorded bird behaviour only when the light treatment (e.g. lasers or spotlights) was applied, assuming that birds did not flee in the absence of a light treatment (Additional file 12: Table S16). Other studies did not include a control treatment for light, but included controls with respect to other variables and tested whether the effect of light on bird behaviour or distribution depends on other factors (e.g. recording the number of birds aggregating at the same light source under varying weather conditions [123]).

\section{Limitations of systematic map}

We strove to find all available evidence and our bibliographic checking confirmed successful realization of that goal for the contexts of bird disorientation, deterrence, and habitat selection. However, we missed about one third of articles that documented aggregation/mortality at illuminated structures. Finding all of the eligible articles indexed in the Web of Science Zoological Record that don't reference light in their topic fields would require screening more than 50,000 additional records, which would delay the publication of this map by many 
months or years. Our systematic search found all references where light was emphasized by the authors as a main contributor to aggregation or mortality in their title, abstract, or keywords. Our search may be biased towards articles that investigated light or assumed light to be a factor contributing to aggregation or mortality, but not articles whose authors considered light irrelevant.

Our search may also have a geographic bias towards North America. We only included articles written in English, which may have contributed to the low number of studies found from South America. Most of our stakeholders were located in North America.

In our Review Findings, we discuss the limitations of the primary evidence base, which limits the potential for further synthesis or evidence-based management. For example, there were 14 bird orders that were never studied, and most studies using white light did not report lamp type or information on spectral composition. Over forty percent of studies in the systematic map lack a control, with this proportion rising to fifty percent in studies relevant to bird deterrence. Many studies reported changes to bird behaviour and distribution, but distribution data were rarely used to quantify species richness or other community outcomes (Table 9).

Where metadata were not possible to code quantitatively or categorically, we described them narratively, and further categorization or unit conversion will be required before conducting a quantitative review or meta-analysis. For example, we categorically coded whether cloud cover/visibility varied within a study, but we narratively reported the cloud cover/visibility values provided by each study's author. These values included proportion cloud cover, visibility distance, clear v. cloudy, and narrative descriptions of how bird behaviour was affected by weather conditions. Lack of detail on weather conditions in many studies limits the potential for reviewing how weather affects bird responses to light. Similarly, we reported light intensity in the units provided by the authors because the variety of units they used (e.g. lux, watts per meter squared, candlepower, candela, nanowatts per square centimetre steridian) are not easily converted to a common unit. The values we reported for light intensity included measurements of brightness, radiance, irradiance, and radiant flux. Further unit conversion and study eligibility screening will be required before comparing bird response across varying light intensities.

Finally, a year has passed between our updated systematic search of the literature and the completion of the map, during which more literature accumulated that we did not assess. The enormous time commitment required of systematic maps and reviews appears to impose a similar limitation on other authors. Creating this database was a substantial effort, involving over 900 hours of work by the authors and an additional 1,500 hours by volunteers. Updating the database would require significant time and funding.

\section{Conclusion}

Our systematic map provides a comprehensive database spanning many human applications of artificial light, countries, bird species, and types of light sources. As scientific research grows exponentially [124], the breadth and depth of human knowledge increases, but finding and comprehending all the available evidence on a broad topic becomes more difficult. The number of search results generated by our search string in the Web of Science Core Collection has nearly doubled over the past 10 years, from approximately 6,500 in 2011 to over 12,500 in 2021. Our inclusive search string and use of specialized databases and websites provided a much more comprehensive evidence base than could be gathered with a basic search of popular databases, such as Google Scholar and Web of Science. Even our own systematic search of these databases found only $75 \%$ of the articles that were ultimately included in this map (Table 3). Our systematic map provides an evidence base that can be searched based on dozens of metadata fields. We hope others will use our systematic map to find evidence relevant to biological and management questions and write reviews, make policy decisions, and target research towards under-studied topics.

\section{Implications for syntheses to inform policy/management}

Using our database, we identified several key evidence clusters with important conservation and management implications that could be prioritized for review. For aggregation and mortality, a subsequent review could study the effects of cloud cover/visibility and lunar phase on bird aggregation and mortality to predict when mortality events are likely and recommend that lights be turned off or dimmed at these times. Another review in this context could identify obstruction lighting characteristics (specifically contrasting red v. white light and flashing v. continuous light) that reduce bird mortality from collisions with tall structures while meeting international standards for aviation safety [88]. For deterrence, the efficacy of red and green lasers could be evaluated in a meta-analysis to provide guidelines for choosing among the most commonly sold lasers. For habitat selection, a review could determine if birds respond differently to white lights produced by halogen, fluorescent, and LED lamps with diverse spectral properties [99]. It may also be possible to review how life history or physiological traits, 
such as foraging strategy or eye-size [13, 114], predict species responses to light (Additional file 16).

\section{Implications for further laboratory or field research}

We also identify knowledge gaps with conservation and management implications that require further laboratory or field research. Considering that light may cause birds to aggregate around structures with artificial lights [28], but can also increase birds' ability to detect and avoid obstacles [54], field studies should investigate whether turning off all light reduces bird mortality at communication towers and other obstructions. The safest lighting options might maximize detectability while minimizing attraction. Choice tests could determine which combinations of wavelengths and flashing rates attract or deter birds, informing efforts to deter birds from zones of human wildlife conflict and reduce collisions. Further research should test deterrents that use UV lights for those bird species that can see in the UV portion of the spectrum. Orientation research should test magnetoreception under artificial light conditions typically experienced by birds in the wild and determine whether artificial light interferes with other orientation mechanisms, especially the stellar compass. We also recommend comparing the effects of different lamp types on bird aggregation/mortality, habitat selection, and orientation. Testing varying spectral properties of LEDs is particularly important to inform lamp choice as older obstruction lighting and streetlamps are replaced [1].

\section{Supplementary Information}

The online version contains supplementary material available at https://doi. org/10.1186/s13750-021-00246-8.

\section{Additional file 1. ROSES form.pdf contains our ROSES form.}

Additional file 2. WOS search strategy.doc is a detailed explanation of our Web of Science search and pre-screening strategies

Additional file 3. WOS Search Records.zip is a compressed folder containing the results of our Web of Science searches. WOS_CC_Long_ String_Search_Results.xIsm is a record of our search in Web of Science Core Collection using the expanded population search string, before any macros were applied.WOS_CC_Short_Search_Results.xlsm is a record of our search in Web of Science Core Collection using the shorter population search string, before any macros were applied. Applying_No_A_or_K Macro.xIsm contains the macros used to identify articles in our Web of Science Core Collection search results indexed without abstracts or keywords and add those search results to the search results that used the short search string, and shows the results of applying those macros. Applying_Replace_Terms_Macro_CC.XIsm contains the macros used to identify articles that only used an intervention/exposure search term in an irrelevant context and shows the results after removing those articles. WOS_CC_for_Rayyan .cSV contains the results of the pre-screened Web of Science Core Collection search, formatted for upload to Rayyan QCRI. WOS_ZR_Search_Results.xIsm is a record of our search in Web of Science Zoological record, before any macros were applied. Applying_Replace Terms_ZR.xlsm contains the macros used to identify articles that only used an intervention/exposure search term in an irrelevant context and shows the results after removing those articles. Applying_Remove_dups_ZRCC. xlsm contains the macros used to remove articles that were found in both the Core Collection and the Zoological Record from the Zoological Record search results. WOS_ZR_for_Rayyan.csv contains the results of the prescreened Web of Science Zoological Record search, formatted for upload to Rayyan QCRI. Documents in the folder Web of Science Core Collection Updated Search and Web of Sciences Zoological Record Updated Search show the equivalent information for the updated searches. Documents in the folder Blank Macro Workbooks are blank macro-enabled workbooks that can be used to apply our macros to any Web of Science Core Collection or Zoological Record search results.

Additional file 4. Database_Search_Records.xls contains several sheets. The first sheet shows the database search records (date of search, screening platform, search string). The other sheets show the articles identified as potentially eligible at the title/abstract screening level, and reasons for exclusion if they were found ineligible at the full text level.A compressed file of PDFs and doc files containing the raw search records is available upon request.

Additional file 5. Website_Search_Records.xls contains three sheets. The first sheet lists the website searched the search string used in Google or the URL of each page within the website that was searched. The second sheet lists the number of eligible articles found on each website. The third sheet lists the URLs of articles found eligible at the title/abstract level, the exclusion reasons for any articles excluded at the full text level, and the citations for any articles found eligible at the full text level.

Additional file 6. Google_Scholar_Search_Results.xls contains multiple sheets. The first sheet shows the combined results from the Google Scholar searches after removing duplicates and formatting them for upload to Rayyan QCRI. The other four sheets show the raw Google Scholar search results, before removal of duplicates.

Additional file 7. Bibliographic_Checking.xls has several sheets. On the READ ME sheet, we explained how we selected reviews for bibliographic checking and describes our scoring system. All_Reviews_From_Rayyan lists all articles found ineligible at the full text level because they were reviews. This sheet also contains the scores assigned to each review. Reviews_for_biblio_checking lists the reviews selected for bibliographic checking. The next 5 sheets list all of the relevant references found in each review and whether or not they were found by our search. The final sheet shows the reasons why some of the references in the review of aggregation/mortality were missed.

Additional file 8. Explanations of Eligibility Criteria.doc provides a detailed explanation or our eligibility criteria.

Additional file 9. Articles_Excluded_at_Full_Text.xls has three sheets. The first lists the articles screened on Rayyan QCRI excluded at the full text level, with reasons for exclusion. The second contains the list of articles screen on other platforms excluded at full text, with reasons for exclusion. The third shows a table of the number of studies excluded for each reason. The fourthlists all articles that were excluded from the map because we could not access their full texts.

Additional file 10. Consistency_Check_Records.xls is the results of the consistency checking exercises for screening at the title/abstract level and the full text level.

Additional file 11. Data_Coding_Fields.xls lists the metadata fields, the codes used in each field, and a description of how the field or codes changed from the protocol. It also lists instructions and notes given to coders. For fields requiring extensive instructions, we list the codes and their instructions on separate sheets.

Additional file 12. Supplementary tables and figures.docx contains all supplementary tables and figures.

Additional file 13. Adams_et_al_2021_Systematic_Map.accdb is the systematic map database for viewing and editing in Microsoft Access.

Additional file 14. Adams_et_al_2021_Systematic_Map_Runtime.accde is the systematic map database for viewing in Microsoft Access or in Microsoft Access Runtime, which can be downloaded for free at https:// 
support.microsoft.com/en-us/office/download-and-install-microsoft365-access-runtime-185c5a32-8ba9-491e-ac76-91 cbe3ea09c9. If opened in Microsoft Access, the user can edit the database and run queries, but not edits or queries will be saved.

Additional file 15. Adams_et_al_2021_Systematic_Map_Excel.xls contains a version of the systematic map in Microsoft Excel

Additional file 16. How_to_use_the_systematic_map_database.doc provides instructions on how to search for studies within the Microsoft Access and Microsoft Excel versions of the database.

\section{Acknowledgements}

In addition to the authors, our mapping team included two members who assisted with the search, consistency checking, and data coding processes: Arden Blumenthal (AB) and Natalia Lifshitz (NL). We thank them for their important contributions to this map. James Adams Campbell generously and patiently helped us create the customized search functions in our Access database. We extend our gratitude to the 25 undergraduate volunteers at who assisted with data coding: Amna Akhtar, Corbin Bishop, Tak Cox, Megan Diep, Shza El Maadawy, Qibang Feng, Saba Gul, Khanh Ha, Emily Halajian, Cala Jorgensen, Jamie Lam, Julia Lee, Fares Mandour, Riya Mangukia, Saad Nizamani, Hima Patel, Jared Randall, Jacquelyn Schenk, Devika Shreekumar, Sigrid Struzik, Kyle Tibayan, Vita Wen, Lucas Wessner, Jacqueline Yang, and Taekwan Yoon. We appreciate the many people and institutions that contributed to training CAA and $A B$ at a systematic evidence synthesis workshops tailored for behavioural ecologists at the Jacob Blaustein Institutes for Desert Research, Ben- Gurion University of the Negev, Israel. The workshop was taught by Biljana Macura (MISTRA-EviEM and Stockholm Environment Institute, Sweden) and organized by Oded Berger-Tal, Alison Greggor, and Daniel Blumstein. The workshop was made possible through collaboration between Ben-Gurion University and Monash University, Australia, as well as generous support from the Jacob Blaustein Center for Scientific Cooperation, The Swiss Institute for Dryland Environmental \& Energy Research, Mitrani Department of Desert Ecology, and Ben-Gurion University of the Negev.

\section{Authors' contributions}

CAA, CCSC, EB, and EFJ collectively identified the topic for the systematic map and developed the protocol. CAA conducted the search and data coding processes, with assistance from a research assistant and volunteers (see acknowledgements). CAA and EB designed the relational database in Microsoft Access. CAA conducted the analyses and wrote the manuscript under the advice of CCSC, EB, and EFJ. All authors read and approved the final manuscript.

\section{Funding}

Graduate student stipends, training in systematic review methodology, and publication costs of the systematic map were funded by NSERC Discovery Grants and Research Fellowships from the Faculty of Science, University of Alberta to CCSC and EB and through the National Fish and Wildlife Foundation and Packard Foundation to EFJ. Graduate student stipends were also provided by the Alberta Student Excellence Scholarship through Alberta Student Aid, the McAfee Estate Scholarship in Zoology, the Computational Biodiversity Science and Services fellowship program, and the Department of Biological Sciences at the University of Alberta.

\section{Availability of data and materials}

All data generated or analysed during this study are included in this published article and its additional files. Where available, article URLs are included in the Systematic Map database.

\section{Declarations}

Ethics approval and consent to participate

Not applicable.

\section{Consent for publication}

Not applicable.

\section{Competing interests}

The authors declare that we have no competing interests.

\section{Author details}

${ }^{1}$ Department of Biological Science, University of Alberta, CW 405, Biological Sciences Building, Edmonton, AB T6G 2E9, Canada. ${ }^{2}$ Department of Biological Sciences, Purdue University, 915 W. State Street, West Lafayette, IN 47907, USA.

Received: 28 June 2021 Accepted: 8 November 2021

Published online: 15 December 2021

\section{References}

1. Kyba CCM, Kuester T, Sánchez de Miguel A, Baugh K, Jechow A, Hölker $\mathrm{F}$, et al. Artificially lit surface of Earth at night increasing in radiance and extent. Sci Adv. 2017;3:e1701528.

2. Sanders D, Frago E, Kehoe R, Patterson C, Gaston KJ. A meta-analysis of biological impacts of artificial light at night. Nat Ecol Evol. 2020. https:// doi.org/10.1038/s41559-020-01322-x.

3. Gaston KJ, Duffy JP, Gaston S, Bennie J, Davies TW. Human alteration of natural light cycles: causes and ecological consequences. Oecologia. 2014;176:917-31.

4. Voigt CC, Roeleke M, Marggraf L, Pētersons G, Voigt-Heucke SL. Migratory bats respond to artificial green light with positive phototaxis. PLoS ONE. 2017;12:e0177748.

5. Kim K-N, Huang Q-Y, Lei C-L. Advances in insect phototaxis and application to pest management: a review. Pest Manag Sci. 2019;75:3135-43.

6. Price JT, Drye B, Domangue RJ, Paladino FV. Exploring the role of artificial lighting in loggerhead turtle (Caretta caretta) nest-site selection and hatchling disorientation. Herpetol Conserv Biol. 2018;13:415-22.

7. Wiltschko W, Munro U, Ford H, Wiltschko R. Red light disrupts magnetic orientation of migratory birds. Nature. 1993;364:525-7.

8. Degen T, Mitesser O, Perkin EK, Weiß N-S, Oehlert M, Mattig E, et al. Street lighting: sex-independent impacts on moth movement. J Anim Ecol. 2016:85:1352-60.

9. McLaren JD, Buler JJ, Schreckengost T, Smolinsky JA, Boone M, Emie van Loon E, et al. Artificial light at night confounds broad-scale habitat use by migrating birds. Ecol Lett. 2018;21:356-64.

10. Stanley TR, White JM, Teel S, Nicholas M. Brightness of the night sky affects loggerhead (Caretta caretta) sea turtle hatchling misorientation but not nest site selection. Front Mar Sci. 2020;7:221.

11. Rodríguez A, Holmes ND, Ryan PG, Wilson K-J, Faulquier L, Murillo Y, et al. Seabird mortality induced by land-based artificial lights. Conserv Biol. 2017;31:986-1001.

12. Ditmer MA, Stoner DC, Francis CD, Barber JR, Forester JD, Choate DM, et al. Artificial nightlight alters the predator-prey dynamics of an apex carnivore. Ecography. 2020. https://doi.org/10.1111/ecog.05251.

13. Santos CD, Miranda AC, Granadeiro JP, Lourenço PM, Saraiva S, Palmeirim JM. Effects of artificial illumination on the nocturnal foraging of waders. Acta Oecologica. 2010;36:166-72.

14. Davies TW, Bennie J, Cruse D, Blumgart D, Inger R, Gaston KJ. Multiple night-time light-emitting diode lighting strategies impact grassland invertebrate assemblages. Glob Change Biol. 2017;23:2641-8.

15. Grubisic M. Waters under artificial lights: does light pollution matter for aquatic primary producers? Limnol Oceanogr Bull. 2018;27:76-81.

16. Hölker F, Wurzbacher C, Weißenborn C, Monaghan MT, Holzhauer SIJ, Premke K. Microbial diversity and community respiration in freshwater sediments influenced by artificial light at night. Phil Trans R Soc B. 2015;370:20140130

17. Yoon TJ, Kim DG, Kim SY, Jo SI, Bae YJ. Light-attraction flight of the giant water bug, Lethocerus deyrolli (Hemiptera: Belostomatidae), an endangered wetland insect in East Asia. Aquat Insects. 2010;32:195-203.

18. Wilson JF, Baker D, Cheney J, Cook M, Ellis M, Freestone R, et al. A role for artificial night-time lighting in long-term changes in populations of 100 widespread macro-moths in UK and Ireland: a citizen-science study. J Insect Conserv. 2018;22:189-96.

19. Boyes DH, Evans DM, Fox R. Is light pollution driving moth population declines? A review of causal mechanisms across the life cycle. Insect Conserv Divers. 2020;14(2):167-87. 
20. Minnaar C, Boyles JG, Minnaar IA, Sole CL, McKechnie AE. Stacking the odds: light pollution may shift the balance in an ancient predator-prey arms race. J Appl Ecol. 2015;52:522-31.

21. Davies TW, Bennie J, Gaston KJ. Street lighting changes the composition of invertebrate communities. Biol Let. 2012;8:764-7.

22. Bennie J, Davies TW, Cruse D, Inger R, Gaston KJ. Cascading effects of artificial light at night: resource-mediated control of herbivores in a grassland ecosystem. Philos Trans R Soc Lond B Biol Sci. 2015. https:// doi.org/10.1098/rstb.2014.0131.

23. Parkinson E, Lawson J, Tiegs SD. Artificial light at night at the terrestrialaquatic interface: effects on predators and fluxes of insect prey. PLoS ONE. 2020;15:e0240138.

24. Knop E, Zoller L, Ryser R, Gerpe C, Hörler M, Fontaine C. Artificial light at night as a new threat to pollination. Nature. 2017. https://doi.org/10. 1038/nature23288.

25. Mann SL, Steidl RJ, Dalton VM. Effects of cave tours on breeding Myotis velifer. J Wildl Manag. 2002;66:618-24.

26. Hori M, Shibuya K, Sato M, Saito Y. Lethal effects of short-wavelength visible light on insects. Sci Rep. 2014;4:7383.

27. Blackwell B F. Understanding avian vision: the key to using light in bird management. In: Vertebrate_Pest_Conference. 2002. Doi:https://doi. org/10.5070/N420110129.

28. Gauthreaux SA Jr, Belser CG. Effects of artificial night lighting on migrating birds. Ecol Conseq Artif Night Light. 2006:67-93.

29. Longcore T, Rich C, Mineau P, MacDonald B, Bert DG, Sullivan LM, et al. Avian mortality at communication towers in the United States and Canada: which species, how many, and where? Biol Cons. 2013;158:410-9.

30. Greene JC. Destruction of migrating birds at the Duluth Ceilometer. Flicker. 1962;34:3-6.

31. Van Doren BM, Horton KG, Dokter AM, Klinck H, Elbin SB, Farnsworth A. High-intensity urban light installation dramatically alters nocturnal bird migration. Proc Natl Acad Sci. 2017;114:11175-80.

32. Loss SR, Will T, Marra PP. Direct mortality of birds from anthropogenic causes. Annu Rev Ecol Evol Syst 2015; 46: 99-120.

33. Troy JR, Holmes ND, Veech JA, Green MC. Using observed seabird fallout records to infer patterns of attraction to artificial light. Endanger Species Res. 2013;22(3):225-34.

34. Graber RR. Nocturnal migration in illinois: different points of view. Wilson Bull. 1968;80:36-71.

35. Larkin RP, Frase BA. Circular paths of birds flying near a broadcasting tower in cloud. J Comp Psychol. 1988;102:90-3.

36. Verheijen F. Photopollution: artificial light optic spatial control systems fail to cope with. Incidents, Causations, Remedies. Exp Biol. 1985:44:1-18.

37. Horváth G, Kriska G, Malik P, Robertson B. Polarized light pollution: a new kind of ecological photopollution. Front Ecol Environ. 2009;7:317-25.

38. Muheim R. Behavioural and physiological mechanisms of polarized light sensitivity in birds. Phil Trans R Soc B. 2011;366:763-71.

39. Chernetsov NS. Orientation and navigation of migrating birds. Biol Bull Russ Acad Sci. 2016:43:788-803.

40. Helbig AJ. Dusk orientation of migratory European robins, Erithacus rubecula: the role of sun-related directional information. Anim Behav. 1991:41:313-22

41. Wiltschko W, Balda RP. Sun compass orientation in seed-caching scrub jays (Aphelocoma coerulescens). J Comp Physiol. 1989;164:717-21.

42. Baldaccini NE, Bezzi EM. Orientational responses to different light stimuli by adult and young sedge warbler (Acrocephalus schoenobaenus) during autumn migration: a funnel technique study. Behaviour. 1989;110:115-24

43. Foster JJ, Smolka J, Nilsson D-E, Dacke M. How animals follow the stars. Proc R Soc B Biol Sci. 2018;285:20172322.

44. Emlen ST. Celestial rotation: its importance in the development of migratory orientation. Science. 1970;170:1198-201.

45. Emlen ST. Migratory orientation in the indigo bunting, Passerina cyanea. Part II: mechanism of celestial orientation. Auk. 1967:84:463-89.

46. Venkatesan A, Lowenthal J, Prem P, Vidaurri M. The impact of satellite constellations on space as an ancestral global commons. Nat Astron. 2020:4:1043-8.

47. Lintott C, Lintott P. Satellite megaclusters could fox night-time migrations. Nature. 2020;586:674-674
48. McDowell JC. The low earth orbit satellite population and impacts of the spaceX starlink constellation. ApJ. 2020;892:L36.

49. Wiltschko R, Wiltschko W. Magnetoreception in birds. J R Soc Interface 2019;16:20190295.

50. Daw N. How vision works: the physiological mechanisms behind what we see. New York: Oxford University Press; 2012.

51. Montràs-Janer T, Knape J, Nilsson L, Tombre I, Pärt T, Månsson J. Relating national levels of crop damage to the abundance of large grazing birds: implications for management. J Appl Ecol. 2019:56:2286-97.

52. Allan JR, Orosz AP. The costs of birdstrikes to commercial aviation. In: Bird strike committee proceedings. Calgary, AB; 2001. p. 10.

53. Frid A, Dill L. Human-caused disturbance stimuli as a form of predation risk. Conserv Ecol. 2002;6. https://www.jstor.org/stable/26271862. Accessed 21 Jan 2021.

54. Blackwell BF, DeVault TL, Fernández-Juricic E, Gese EM, Gilbert-Norton L, Breck SW. No single solution: application of behavioural principles in mitigating human-wildlife conflict. Anim Behav. 2016;120:245-54.

55. Bradley, F. Blackwell, Fernandez-Juricic E. Behavior and physiology in the development and application of visual deterrents at airports. In: Wildlife in airport environments: preventing animal- aircraft collisions through science-based management. 2013. p. 11-22.

56. Hutto R. Habitat selection by nonbreeding, migratory land birds. In: Habitat selection in birds. 1985. p. 455-76.

57. Johnson MD. Measuring habitat quality: a review. The Condor. 2007;109:489.

58. Jones J. Habitat selection studies in avian ecology: a critical review. Auk 2001:118:557-62.

59. Amichai E, Kronfeld-Schor N. Artificial Light at night promotes activity throughout the night in nesting common swifts (Apus apus). Sci Rep. 2019;9:11052.

60. Dominoni DM, Partecke J. Does light pollution alter daylength? A test using light loggers on free-ranging European blackbirds (Turdus merula ). Phil Trans R Soc B. 2015;370:20140118.

61. Zhang $X$, Yang W, Liang W, Wang Y, Zhang S. Intensity dependent disruptive effects of light at night on activation of the HPG axis of tree sparrows (Passer montanus). Environ Pollut. 2019;249:904-9.

62. Moaraf S, Vistoropsky Y, Pozner T, Heiblum R, Okuliarová M, Zeman M, et al. Artificial light at night affects brain plasticity and melatonin in birds. Neurosci Lett. 2020;716:134639.

63. Taufique ST, Prabhat A, Kumar V. Light at night affects hippocampal and nidopallial cytoarchitecture: implication for impairment of brain function in diurnal corvids. J Exp Zool A Ecol Integr Physiol. 2019;331:149-56

64. Raap T, Casasole G, Pinxten R, Eens M. Early life exposure to artificial light at night affects the physiological condition: an experimental study on the ecophysiology of free-living nestling songbirds. Environ Pollut. 2016:218:909-14.

65. Raap T, Casasole G, Costantini D, AbdElgawad H, Asard H, Pinxten R, et al. Artificial light at night affects body mass but not oxidative status in free-living nestling songbirds: an experimental study. Sci Rep. 2016. https://doi.org/10.1038/srep35626.

66. Grunst ML, Raap T, Grunst AS, Pinxten R, Parenteau C, Angelier F, et al. Early-life exposure to artificial light at night elevates physiological stress in free-living songbirds tr. Environ Pollut. 2020;259:113895.

67. Owens ACS, Lewis SM. The impact of artificial light at night on nocturnal insects: a review and synthesis. Ecol Evol. 2018;8:11337-58.

68. Borchard P, Eldridge DJ. Does artificial light influence the activity of vertebrates beneath rural buildings? Aust J Zool. 2013;61:424

69. Canário F, Hespanhol Leitão A, Tomé R. Predation Attempts by shorteared and long-eared owls on migrating songbirds attracted to artificial lights. J Raptor Res. 2012;46:232-4.

70. Bock CE, Jones ZF. Avian habitat evaluation: should counting birds count? Front Ecol Environ. 2004;2:403-10.

71. Gilroy J, Sutherland W. Beyond ecological traps: perceptual errors and undervalued resources. Trends Ecol Evol. 2007;22:351-6.

72. Gehring J, Kerlinger P, Manville AM. Communication towers, lights, and birds: successful methods of reducing the frequency of avian collisions. Ecol Appl. 2009;19:505-14.

73. Poot H, Ens BJ, de Vries H, Donners MAH, Wernand MR, Marquenie JM Green light for nocturnally migrating birds. Ecol Soc. 2008. https://doi org/10.5751/ES-02720-130247. 
74. Cassidy FL. The potential of lasers as deterrents to protect birds in the Alberta oil sands and other areas of human-bird conflict. University of Alberta; 2015.

75. Andelt WF, Woolley TP, Hopper SN. Effectiveness of barriers, pyrotechnics, flashing lights, and scarey man for deterring heron predation on fish. Wildl Soc Bull. 1997;25:686-94.

76. Blackwell BF, DeVault TL, Seamans TW, Lima SL, Baumhardt P, Fernández-Juricic E. Exploiting avian vision with aircraft lighting to reduce bird strikes: exploiting avian vision with aircraft lighting. J Appl Ecol. 2012;49:758-66.

77. de Molenaar JG, Sanders ME, Jonkers DA. Road Lighting and Grassland Birds: Local Influence of Road Lighting on a Black-Tailed Godwit Population. In: Ecological consequences of artificial night lighting. Island Press; 2006. p. 114-36.

78. Arai M, Nakajima K, Arai Y, Kondo T. Impact of illumination of long marine bridges on birds in surrounding strait area. 2004. p. 719-24. https://ieeexplore.ieee.org/abstract/document/1405522.

79. Gorenzel P, Salmon T. Bird hazing manual: techniques and strategies for dispersing birds from spill sites. UNCANR Publications; 2008.

80. Adams CA, Blumenthal A, Fernández-Juricic E, Bayne E, St. Clair CC. Effect of anthropogenic light on bird movement, habitat selection, and distribution: a systematic map protocol. Environ Evid. 2019:8:13.

81. Pullin AS, Frampton GK, Livoreil B, Petrokofsky G. Guidelines for systematic reviews in environmental management version 5.0. collaboration for environmental evidence; 2018. www.environmentalevidence.org/ information-for-authors.

82. Haddaway NR, Macura B, Whaley P, Pullin AS. ROSES Reporting standards for Systematic Evidence Syntheses: pro forma, flow-diagram and descriptive summary of the plan and conduct of environmental systematic reviews and systematic maps. Environ Evid. 2018;7:7.

83. Harzing AW. Publish or perish 6. https://harzing.com/resources/publi sh-or-perish.

84. Rayyan QCRI. https://rayyan.qcri.org/users/sign_in. Accessed 6 Aug 2019

85. Gilsdorf JM, Hygnstrom SE, VerCauteren KC. Use of frightening devices in wildlife damage management. Integr Pet Manage Rev. 2002;7(1):29-45

86. Wiltschko R, Stapput K, Thalau P, Wiltschko W. Directional orientation of birds by the magnetic field under different light conditions. J R Soc Interface. 2010;7(Suppl_2):S163-77.

87. Leveau LM. Urbanization, environmental stabilization and temporal persistence of bird species: a view from Latin America. Peer J. 2018:6:e6056.

88. Lele SR, Merrill EH, Keim J, Boyce MS. Selection, use, choice and occupancy: clarifying concepts in resource selection studies. J Anim Ecol. 2013;82:1183-91.

89. Donato DB, Nichols O, Possingham H, Moore M, Ricci PF, Noller BN. A critical review of the effects of gold cyanide-bearing tailings solutions on wildlife. Environ Int. 2007;33:974-84.

90. Cook A, Rushton S, Allan J, Baxter A. An evaluation of techniques to control problem bird species on landfill sites. Environ Manage. 2008:41:834-43.

91. Microsoft Access 2016 Runtime. Microsoft Download Center. https:// www.microsoft.com/en-ca/download/details.aspx?id=50040. Accessed 27 Oct 2021.

92. Orders of Birds_-IOC World Bird List. https://www.worldbirdnames.org/ new/classification/orders-of-birds-draft/. Accessed 29 Apr 2021.

93. Calvert AM, Walde SJ, Taylor PD. Nonbreeding-season drivers of population dynamics in seasonal migrants: conservation parallels across taxa. 2009. https://DalSpace.library.dal.ca//handle/10222/29831. Accessed 7 Mar 2021

94. Seymoure BM, Linares C, White J. Connecting spectral radiometry of anthropogenic light sources to the visual ecology of organisms. J Zool. 2019;308:93-110.

95. Inger R, Bennie J, Davies TW, Gaston KJ. Potential biological and ecological effects of flickering artificial light. PLoS ONE. 2014;9:e98631.

96. Kelber A. Bird colour vision — from cones to perception. Curr Opin Behav Sci. 2019;30:34-40.

97. Goller B, Blackwell BF, DeVault TL, Baumhardt PE, Fernández-Juricic E. Assessing bird avoidance of high-contrast lights using a choice test approach: implications for reducing human-induced avian mortality. Peer J. 2018:6:e5404
98. Briot JL. Last experiments with a laser equipment designed for Avian dispersal in airport environment. Paper presented at the International Bird Strike Committee. Retrieved 24th August, 2007. http://www.intbi rdstrike.org/Athens_Papers/IBSC27\%20WPV-1.pdf. 2005.

99. Elvidge CD, Keith DM, Tuttle BT, Baugh KE. Spectral identification of lighting type and character. Sensors. 2010;10:3961-88.

100. Kyba CCM, Conrad J, Shatwell T. Lunar illuminated fraction is a poor proxy for moonlight exposure. Nat Ecol Evol. 2020. https://doi.org/10. 1038/s41559-020-1096-7.

101. Patterson JW. Evaluation of new obstruction lighting techniques to reduce avian fatalities. 2012. http://www.tc.faa.gov/its/worldpac/techr pt/tctn12-9.pdf.

102. Guidelines for lighting and marking of structures supporting renewable energy development. Bureau of Ocean Energy Management; 2021. https://www.boem.gov/sites/default/files/documents/renewableenergy/2021-Lighting-and-Marking-Guidelines.pdf.

103. International standards and recommended practices. Annex 14 to the Convention on International Civil Aviation. Volume 1: aerodome design and operations. Chapter 6. Visual aids for denoting obstacles. International Civil Aviation Organization; 2013. https://www.wetraobstructio nlight.com/wp-content/uploads/2017/04/ICAO-Annex-14.pdf.

104. Dwyer JF, Pandey AK, McHale LA, Harness RE. Near-ultraviolet light reduced Sandhill Crane collisions with a power line by $98 \%$. The Condor. 2019;121:duz008.

105. Patterson J. Performance Assessment of the Vestas InteliLight ${ }^{\mathrm{TM}}$ X-Band System as an Aircraft Detection Lighting System (ADLS). Federal Aviation Administration; 2018. http://www.tc.faa.gov/its/worldpac/techrpt/ tc18-22.pdf

106. May R, Reitan O, Bevanger K, Lorentsen S-H, Nygård T. Mitigating windturbine induced avian mortality: sensory, aerodynamic and cognitive constraints and options. Renew Sustain Energy Rev. 2015:42:170-81.

107. Manly BF, McDonald L, Thomas DL, McDonald TL, Erickson WP. Resource selection by animals: statistical design and analysis for field studies. Springer Science \& Business Media; 2007

108. Wiltschko R, Dehe L, Gehring D, Thalau P, Wiltschko W. Interactions between the visual and the magnetoreception system: different effects of bichromatic light regimes on the directional behavior of migratory birds. J Physiol Paris. 2013;107:137-46.

109. Emlen ST, Emlen JT. A technique for recording migratory orientation of captive birds. Auk. 1966:83:361-7.

110. Mangel JC, Wang J, Alfaro-Shigueto J, Pingo S, Jimenez A, Carvalho F, et al. Illuminating gillnets to save seabirds and the potential for multitaxa bycatch mitigation. R Soc open sci. 2018;5:180254.

111. Bielli A, Alfaro-Shigueto J, Doherty PD, Godley BJ, Ortiz C, Pasara A, et al. An illuminating idea to reduce bycatch in the Peruvian small-scale gillnet fishery. Biol Conserv. 2020;241:108277.

112. Field R, Crawford R, Enever R, Linkowski T, Martin G, Morkūnas J, et al. High contrast panels and lights do not reduce bird bycatch in Baltic Sea gillnet fisheries. Glob Ecol Conserv. 2019;18:e00602.

113. Martin GR. Understanding bird collisions with man-made objects: a sensory ecology approach. Ibis. 2011;153:239-54.

114. Ausprey IJ, Newell FL, Robinson SK. Adaptations to light predict the foraging niche and disassembly of avian communities in tropical countrysides. Ecology. 2021;102:e03213.

115. Suzuki K, Ikebuchi M, Okanoya K. The impact of domestication on fearfulness: a comparison of tonic immobility reactions in wild and domesticated finches. Behav Proc. 2013;100:58-63.

116. Longcore T, Rodríguez A, Witherington B, Penniman JF, Herf L, Herf M. Rapid assessment of lamp spectrum to quantify ecological effects of light at night. J Exp Zool A Ecol Integr Physiol. 2018;329:511-21.

117. Longcore T, Aldern HL, Eggers JF, Flores S, Franco L, Hirshfield-Yamanishi $E$, et al. Tuning the white light spectrum of light emitting diode lamps to reduce attraction of nocturnal arthropods. Phil Trans R Soc B. 2015:370:20140125

118. Plummer KE, Hale JD, O'Callaghan MJ, Sadler JP, Siriwardena GM. Investigating the impact of street lighting changes on garden moth communities. J Urban Ecol. 2016. https://doi.org/10.1093/jue/juw004.

119. Jaggard AK, Smith N, Torpy FR, Munro U. Rules of the roost: characteristics of nocturnal communal roosts of rainbow lorikeets (Trichoglossus haematodus, Psittacidae) in an urban environment. Urban Ecosyst. 2015:18:489-502. 
120. Daoud-Opit S, Jones DN. Guided by the light: roost choice and behaviour of urban Rainbow Lorikeets (Trichoglossus haematodus). Eur J Ecol. 2016;2:72-80.

121. Gorenzel WP, Salmon TP. Characteristics of American crow urban roosts in California. J Wildl Manag. 1995;59:638-45.

122. van Tets GF, Vestjens WJM, Slater E. Orange runway lighting as a method for reducing bird strike damage to aircraft. CSIRO Wildl Res. 1969;14:129-51.

123. Hüppop O, Hilgerloh G. Flight call rates of migrating thrushes: effects of wind conditions, humidity and time of day at an illuminated offshore platform. J Avian Biol. 2012;43:85-90.

124. Parolo PDB, Pan RK, Ghosh R, Huberman BA, Kaski K, Fortunato S. Attention decay in science. J Informet. 2015;9:734-45.

\section{Publisher's Note}

Springer Nature remains neutral with regard to jurisdictional claims in published maps and institutional affiliations.

- fast, convenient online submission

- thorough peer review by experienced researchers in your field

- rapid publication on acceptance

- support for research data, including large and complex data types

- gold Open Access which fosters wider collaboration and increased citations

- maximum visibility for your research: over $100 \mathrm{M}$ website views per year

At BMC, research is always in progress.

Learn more biomedcentral.com/submissions 